OPEN ACCESS

Edited by:

Nicoletta Pedemonte,

Istituto Giannina Gaslini (IRCCS), Italy

Reviewed by:

Anna Cereseto,

University of Trento, Italy

Agnieszka Swiatecka-Urban,

University of Pittsburgh, United States

*Correspondence:

Isabelle Sermet-Gaudelus

isabelle.sermet@aphp.fr

Specialty section:

This article was submitted to

Pharmacology of lon Channels

and Channelopathies,

a section of the journal

Frontiers in Pharmacology

Received: 02 October 2018

Accepted: 31 January 2019

Published: 27 February 2019

Citation:

Pranke I, Golec A, Hinzpeter A,

Edelman $A$ and Sermet-Gaudelus I

(2019) Emerging Therapeutic Approaches for Cystic Fibrosis. From

Gene Editing to Personalized Medicine. Front. Pharmacol. 10:121.

doi: 10.3389/fphar.2019.00121

\section{Emerging Therapeutic Approaches for Cystic Fibrosis. From Gene Editing to Personalized Medicine}

\author{
Iwona Pranke1, Anita Golec ${ }^{1}$, Alexandre Hinzpeter ${ }^{1}$, Aleksander Edelman' ${ }^{1}$ and \\ Isabelle Sermet-Gaudelus 1,2,3*
}

${ }^{1}$ INSERM U 1151, Institut Necker Enfants Malades, Université Paris Descartes, Paris, France, ${ }^{2}$ Centre de Référence Maladie Rare, Mucoviscidose et Maladies de CFTR, Paris, France, ${ }^{3}$ Faculté de Médecine, Université Paris Descartes, Paris, France

An improved understanding of the cystic fibrosis (CF) transmembrane conductance regulator (CFTR) protein structure and the consequences of CFTR gene mutations have allowed the development of novel therapies targeting specific defects underlying CF. Some strategies are mutation specific and have already reached clinical development; some strategies include a read-through of the specific premature termination codons (read-through therapies, nonsense mediated decay pathway inhibitors for Class I mutations); correction of CFTR folding and trafficking to the apical plasma membrane (correctors for Class II mutations); and an increase in the function of CFTR channel (potentiators therapy for Class III mutations and any mutant with a residual function located at the membrane). Other therapies that are in preclinical development are not mutation specific and include gene therapy to edit the genome and stem cell therapy to repair the airway tissue. These strategies that are directed at the basic CF defects are now revolutionizing the treatment for patients and should positively impact their survival rates.

Keywords: cystic fibrosis, CFTR, ivacaftor, CFTR modulator, gene therapy

\section{INTRODUCTION}

Cystic fibrosis (CF) is an autosomal recessive disease that affects approximately 75,000 people in North America, Europe, and Australia alone. The life expectancy of CF patients has been constantly increasing because of symptomatic therapies. As our knowledge of the CF transmembrane conductance regulator (CFTR) structure and the functional consequences of its mutations has improved, therapeutics to restore CFTR expression and function have begun to emerge. Search for mutation-specific and mutation-independent tactics have now opened the path toward a revolutionizing approach in treating CF patients.

\section{BACKGROUND}

\section{CFTR Biology and Cystic Fibrosis}

The CFTR is a transmembrane chloride $\left(\mathrm{Cl}^{-}\right)$and bicarbonate $\left(\mathrm{HCO}_{3}{ }^{-}\right)$ion channel that is expressed in the apical membranes of the epithelial cells of multiple exocrine organs, where it regulates salt and fluid homeostasis (Linsdell, 2014). The CFTR glycoprotein has 
multiple membrane-integrated subunits that form two membrane-spanning domains (MSD), two nucleotide-binding domains (NBD), and a regulatory (R) domain that acts as a phosphorylation site (Rowe et al., 2005; Riordan, 2008). MSD1 and MSD2 form the walls of the channel pore, and their conformational changes drive the channel's opening and closing (Muallem and Vergani, 2009). Phosphorylation of the R domain, which is driven by cAMP-dependent protein kinase $\mathrm{A}$ and $\mathrm{C}$, enhances adenosine triphosphate (ATP) association to the NBD domains and hence mediates their conformational change and their dimerization in a head-to-tail configuration. This conformation defines the open state of the channel (Riordan, 2008). Inversely, the hydrolysis of ATP drives the channel to a basal closed state (Vergani et al., 2005).

CFTR protein maturation, which is characterized by complex domain folding, assembly, and double $N$-glycosylation of the MSD2, starts early in the endoplasmic reticulum (ER) during translation and continues in the Golgi apparatus. This complex process can be mismatched and retarded at multiple steps, leading to important (60-80\%) degradation of even wild-type CFTR (WT-CFTR) (Varga et al., 2004) by the ER-associated ubiquitin-dependent degradation system (Jensen et al., 1995; Gelman et al., 2002) or autophagy. After reaching the plasma membrane (PM), the WT-CFTR channel is internalized by clathrin-dependent endocytosis and recycles back to the cell surface through recycling endosomes. Because the mature WTCFTR is very stable at the PM, a pool of $10 \%$ WT-CFTR is internalized and recycled back to the PM each minute (Sharma et al., 2004; Pranke and Sermet-Gaudelus, 2014).

Almost 2,000 mutations in the CFTR gene have been found to cause CF; they decrease the flow of $\mathrm{Cl}^{-}$and $\mathrm{HCO}_{3}{ }^{-}$ through the epithelia of multiple organs, including the lung, pancreas, sweat glands, vas deferens, liver, and intestine. As a consequence, they interfere with their normal functioning. In the respiratory tracts, the lack of a CFTR function drives the accumulation of abnormally thick and sticky mucus that underlies chronic lung inflammation and recurrent bacterial infections, leading to progressive lung degradation. There is increasing evidence that airway inflammation and infection are frequently present before the appearance of symptoms; however, it is not clear which comes first. Studies on CF animal models suggest that CF causes congenital airway abnormalities, such as a narrowed trachea and that the airway surface liquid has a reduced $\mathrm{pH}$ level in $\mathrm{CF}$, leading to an impaired bacteriakilling potential. The accumulation of mucus gives rise to sterile inflammation. These pathological conditions initiate a vicious circle that leads to bronchial wall inflammation and air trapping. The cumulation of neutrophils further enhances inflammation through the production of elastase and proinflammatory cytokines (Nichols and Chmiel, 2015). This ultimately leads to the formation of bronchiectasis. Lung disease is responsible for $>95 \%$ of CF deaths (Davis et al., 1996; Stoltz et al., 2015). Currently, according to the CF foundation registry, the median survival age of those born in 2016 is predicted to be 47.7 years of age (Cystic Fibrosis Registry 2016). Because the morbidity and mortality of CF patients is mainly caused by lung disease, the principal focus of research in the
CF domain and therapy development has been targeted at minimizing lung disease.

\section{Mutations Inducing CF}

CFTR mutations are divided into six classes determined by the specific defect in CFTR protein synthesis, trafficking, function, or stability (O'Sullivan and Freedman, 2009) (Figure 1 and Table 1), although many CFTR mutants present multiple defects, such as F508del-CFTR with deficient trafficking, function, and stability (Veit et al., 2016).

Class I mutations lead to severely defective protein production. They are primarily nonsense or frameshift mutations introducing a premature termination codon (PTC), leading to unstable messenger RNA (mRNA) degraded by the mRNA decay pathway (NMD) (Maquat, 2004; Popp and Maquat, 2016; Martiniano et al., 2016). If the mRNA is translated, PTC decoding results in ribosome disruption and premature translation termination, usually resulting in synthesis of a nonfunctional, shortened CFTR protein. Large insertions/deletions and splicing mutations resulting in the absence of proteins at the $\mathrm{PM}$ are also included in Class I.

Class II mutations introduce defects in CFTR processing. The main example of this class is the in-frame deletion of the 508 amino acid phenylalanine (p.Phe508del, legacy name F508del) which affects about $80 \%$ of patients. Defective folding of the newly synthesized F508del-CFTR renders NBD1 instable energetically and impairs the assembly of the interface between NBD1 and MSD1/MSD2 (Du et al., 2005; Billet et al., 2013). This misfolding impairs its stability at the ER, promoting premature degradation by the ubiquitin-dependent proteasome (Lukacs and Verkman, 2012). Consequently, little or no CFTR is trafficked to the apical PM. This mutation is also associated with the impairment of $\mathrm{Cl}^{-}$channel gating and decreased CFTR stability at the PM because of increased degradation by a peripheral ubiquitination-related protein quality control system (Lukacs et al., 1993).

Class III mutations produce CFTR protein localizing at the PM but with defective activation, leading to a severe decrease in the ion channel's open probability. The c.1652G > A mutation, legacy name G551D (located in exon 11), affects about 2-4\% of patients and is the most frequent in this class. In this case, the substitution of the amino acid glycine by aspartate occurs at a crucial point in the NBD1-NBD2 interface, inactivates ATPdependent gating, and decreases open probability by $\sim 100$-fold compared with WT-CFTR.

Class IV mutations induce channel dysfunction by defective ion conductance; these mutations mostly involve the MSD regions of the CFTR protein, forming the pore of the channel. The Class IV missense mutations provide a protein located in the apical membrane but with only the residual activity of a cAMP-dependent $\mathrm{Cl}^{-}$secretion. The most common Class IV mutation is c.350G > A, legacy name R117H (located in exon 4), which affects $0.7 \%$ of patients. This substitution of arginine with histidine at position 117, which is located in the region of TMD2, reduces the channel open probability by $75 \%$, and changes $\mathrm{Cl}^{-}$ and $\mathrm{HCO}_{3}{ }^{-}$conductance (LaRusch et al., 2014). Because Class IV mutations lead to the biosynthesis of CFTR retaining residual 


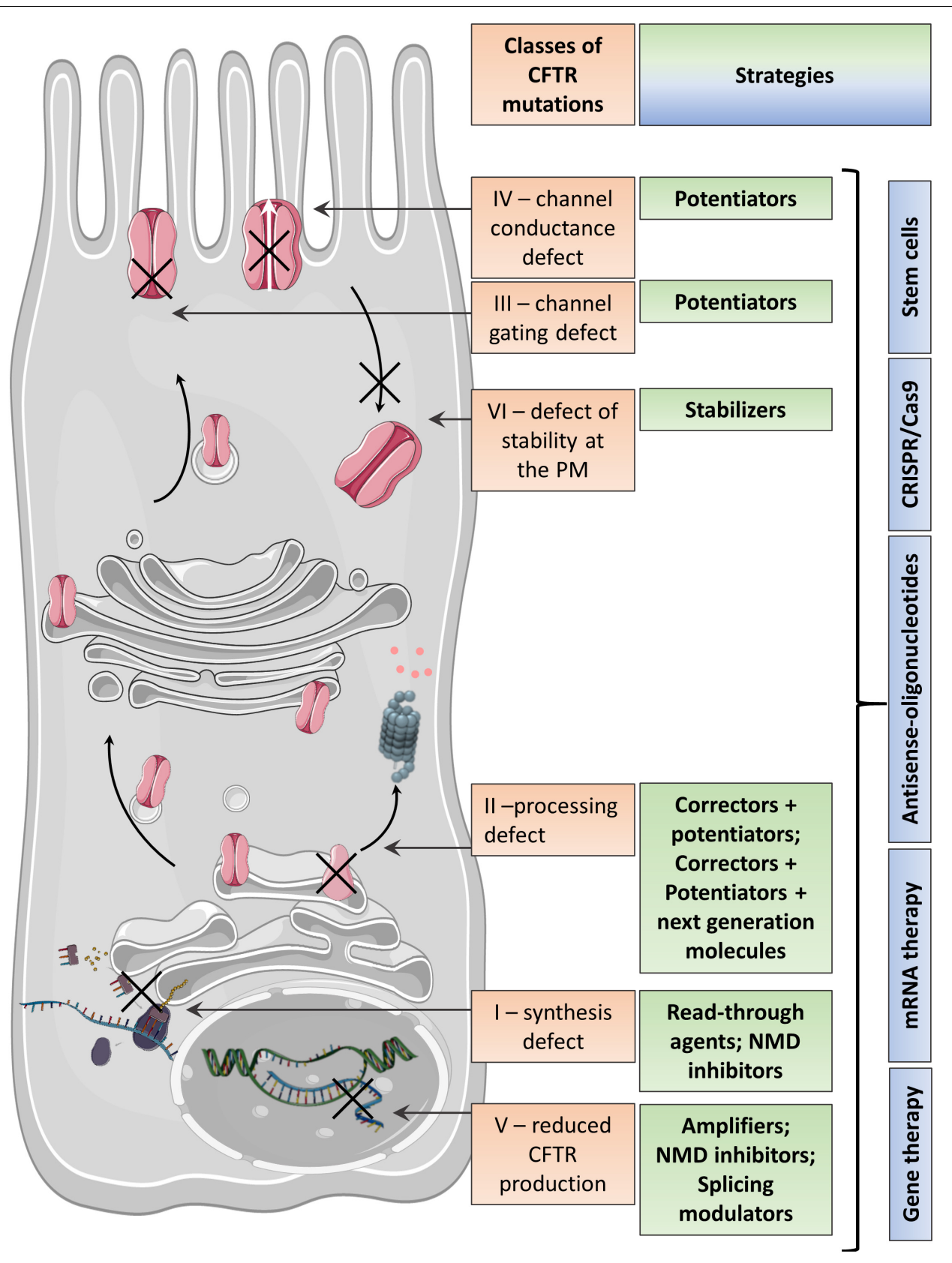

FIGURE 1 | Variable CFTR protein defects causing CF disease and the corresponding therapeutic strategies.

function and normal regulation, simple therapies to improve their activity are efficient.

Class $\mathrm{V}$ mutations are characterized by reduced amounts of normally functioning CFTR at the apical PM. Most of these mutations affect pre-mRNA splicing. This induces complete or partial exclusion of the exon, generating missense, silent, or nonsense mutations and, consequently, the production of defective CFTR. Class V mutations are either intronic mutations inducing the incorporation of cryptic exons or exonic mutations altering splicing enhancer motifs. The most common mutation from this class is c.3718-2477C $>\mathrm{T}$, legacy name $3849+10 \mathrm{~kb} \mathrm{C} \rightarrow \mathrm{T}$ (located in intron 19), and affecting about $0.58 \%$ of patients but with a higher frequency in specific populations, such as Ashkenazi Jews.

Class VI mutations result in the decreased stability of CFTR at the apical membrane as a result of increased endocytosis or decreased recycling to the PM. An example of Class VI mutations is c.120del23. This deletion of nucleotides 120 up to 142 in exon 1 eliminates the translation initiation codon at nucleotides 133-135, and the translation instead initiates at sites in exon 4 at M150/M152/M156. This produces N-truncated 
proteins that are unstable and display reduced $\mathrm{Cl}^{-}$channel activity (Ramalho et al., 2009).

Although mutations of Classes I-III provoke more severe CF disease with absent or very weak residual CFTR activity, mutations representing Classes IV-VI lead to relatively high residual function and are associated with milder forms of CF.

\section{Different Treatment Strategies}

Some strategies are specific to the CFTR mutation and aim to (i) bypass a specific PTC and restore mRNA levels (read-through therapies, NMD inhibitors therapy for Class I mutations), (ii) correct CFTR folding and trafficking to the apical PM (correctors for Class II mutations), or (iii) increase the CFTR channel function (potentiators therapy for Class III mutations and any mutant with residual function located at the PM) (Figure $\mathbf{1}$ and Table 1). Other therapies in preclinical development are not mutation specific and include gene therapy to edit the genome and stem cell therapy to repair the airway tissue.

The personalization of therapy for a given patient is based on the paradigm of selecting the most effective molecule or association of molecules. The functional assays that directly or indirectly measure the CFTR activity in in vitro cultures of primary nasal epithelial cells (Pranke et al., 2017) and the cultures of organoids developed from intestinal epithelia (Dekkers et al., 2013, 2016) and nasal/bronchial spheroids (Brewington et al., 2018; Guimbellot et al., 2018) are promising tools to use as patientspecific biomarkers, predictive of clinical efficacy of these novel therapies.

\section{GENETIC THERAPIES}

Cystic fibrosis genetic therapies rely on delivering DNA or RNA, which encodes the CFTR protein or on the restoration of the CFTR gene (genome editing) or the CFTR mRNA (mRNA editing).

\section{Gene Therapy}

Gene therapy implies the relocation of the proper copies of the CFTR gene to the epithelial cell layer in the airways with the goal of replacing the mutated gene and express functional CFTR protein. For high efficiency of this therapy, DNA coding for CFTR together with regulatory components must be adequately administered to the airways, reach the target cells, enter (transduce) the cell, and express CFTR protein. Because CF is a monogenic disease, gene therapy is particularly attractive. Despite the fact that $\mathrm{CF}$ is a multiorgan disease, improving respiratory manifestations will lead to a significant improvement in the patient's quality of life and may be associated with a decrease in mortality. The inhaled route is the easiest way to access the targeted abnormal zones.

Although gene therapy carries promise, it has several limitations. First, finding the appropriate plasmid DNA molecule model is important in terms of clinical potency (Pringle et al., 2009; Dhand, 2017). Second, natural barriers such as mucus, versatile immune responses, and intracellular limitations considerably impair gene transfer into the lungs (Osman et al., 2018). Finally, because the airway epithelium is constantly renewing, genetic therapies necessitate repeated administration. Therefore, the selection of the appropriate delivery method is

TABLE 1 | Classification of CFTR mutations.

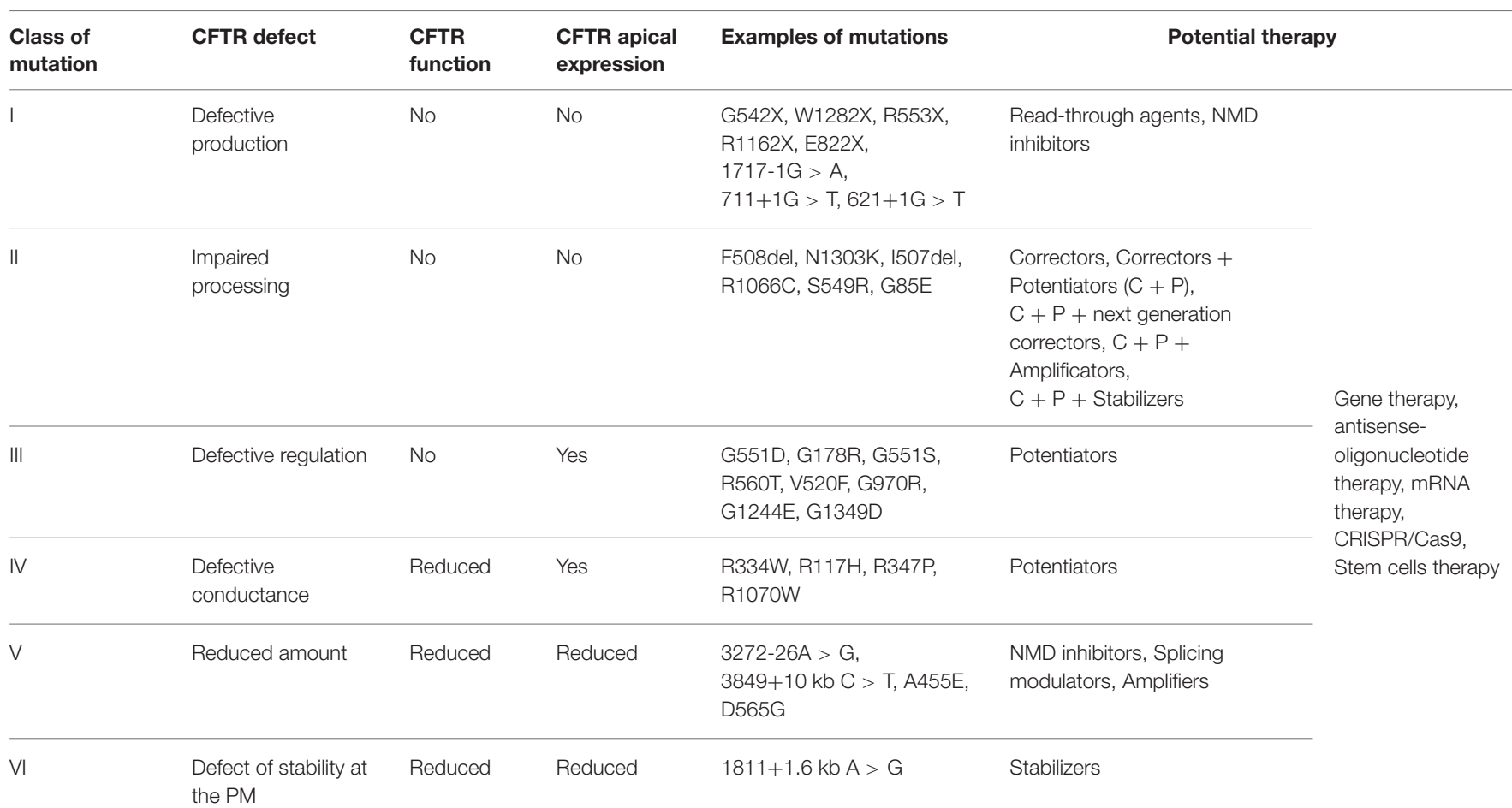



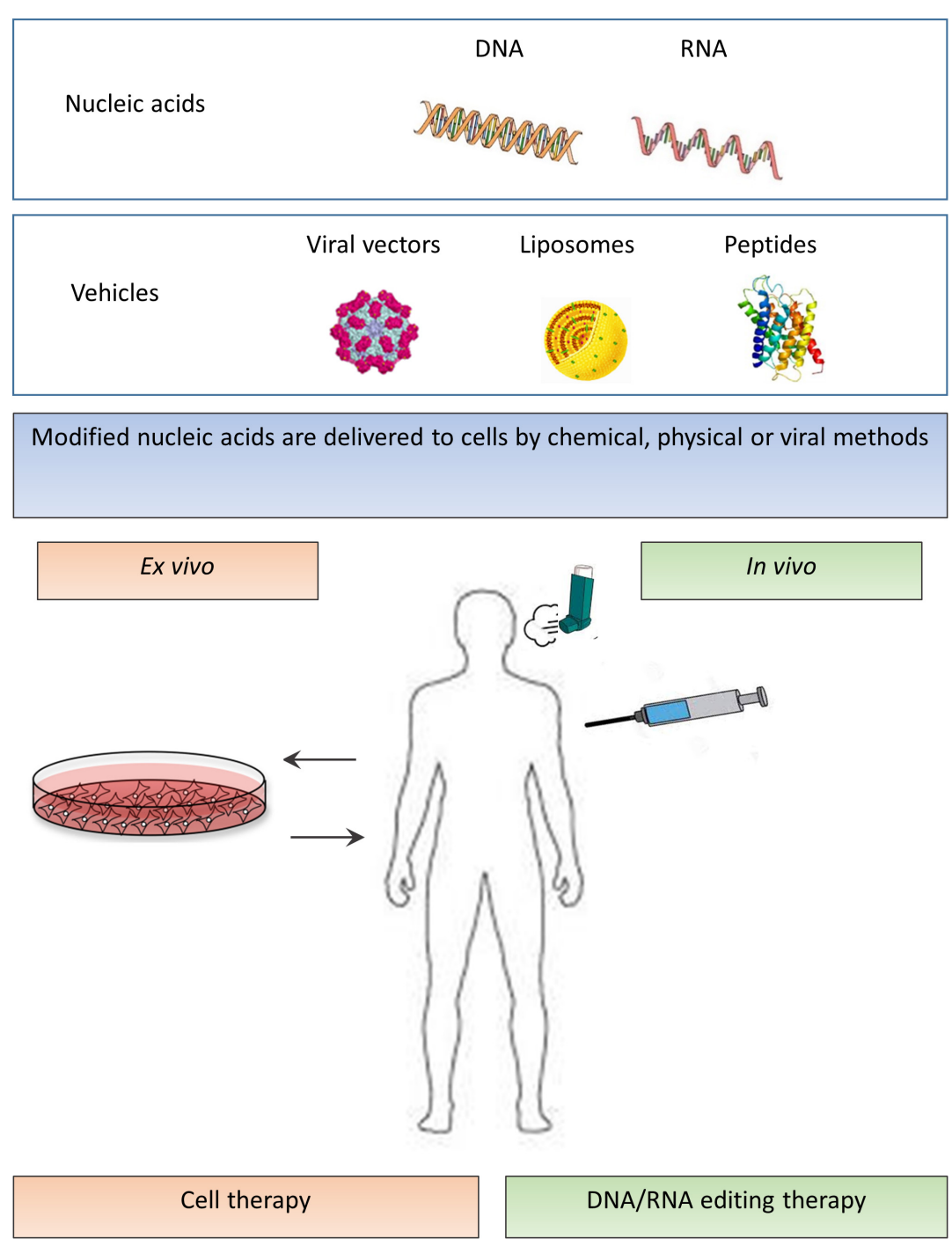

FIGURE 2 | In vivo and ex vivo strategies of mutation independent approaches. Modified nucleic acids (upper panel - nucleic acids) are delivered to cells by various methods (second upper panel - vehicles). In ex vivo cell therapy, stem or progenitor cells are derived from the subject, and after the required modification, the cells are transferred back into the patient (lower left part of the panel). In in vivo DNA/RNA editing therapy, a direct transfer of genes to the patient is performed by viral or non-viral methods (lower right part of the panel).

essential. The most commonly used agents in gene therapy for CF are viral vectors: adenoviruses, adeno-associated viruses, and lentiviruses, but also non-viral lipoplexes and peptide nanoparticles (Figure 2).

In 1990, for the first time, Drumm et al. (1990) proved that it is possible to deliver a healthy CFTR gene into the adenocarcinoma cell of a CF patient by means of retrovirus transduction. Expression of a normal CFTR gene was linked to a cAMP-dependent $\mathrm{Cl}^{-}$channel regulation in CF epithelial cells.

The first ever use of viral and subsequent non-viral gene transfer factors on nasal and bronchial epithelium was seen in clinical trials in 1993 (Zabner et al., 1993; Crystal et al., 1994; Caplen et al., 1995). Many subsequent trials have demonstrated evidence of CFTR expression but have not achieved clinical efficacy.

\section{Viral Vectors-Adenoviruses}

Rosenfeld et al. (1992) showed that the adenovirus-mediated transfer of DNA coding for human CFTR to a cotton-rat model by intratracheal introduction resulted in mRNA and functional protein expression. In turn, Zabner et al. (1993) performed adenoviral gene therapy tests in humans. Although neither the CFTR mRNA nor the protein were detectable after a single nasal application of the CFTR gene-containing vector, the nasal potential difference showed a limited improvement of the conductivity of the $\mathrm{Cl}^{-}$channel. Crystal et al. (1994) was the first to detect CFTR protein in the lung and nose tissue after adenoviral vector administration. Additional surveys on gene transfer by adenovirus transduction of the nasal epithelium proved that the expression of CFTR mRNA and/or CFTR protein is transient and that the $\mathrm{Cl}^{-}$transport was not fully recovered, as 
shown by the nasal potential difference (Hay et al., 1995; Knowles et al., 1995; Zabner et al., 1996; Bellon et al., 1997).

Despite preliminary promising data from preclinical models of nasal and pulmonary tissues (Katkin et al., 1995; Scaria et al., 1998) and a good tolerance at low-to-intermediate doses (Harvey et al., 2002), adenovirus-mediated gene transfer proved inefficient in CF patients (Joseph et al., 2001). This mostly occurred because of the lack of the coxsackie-adenovirus receptor, which is absent from the apical surface of most human airway epithelial cells (HAE) (Walters et al., 1999). The transduction efficiency was increased by a tight junction opener (Gregory et al., 2003) and the association of the adenovirus to the 2-(diethylamino) ethyl ether (DEAE) dextran. However, clinical use of these tight junction openers could introduce the risk of systemic invasion when considering the significant presence of bacteria in the CF lung.

\section{Adeno-Associated Virus (AAV) Vectors}

The substantial achievement in gene therapy by using rAAV for congenital blindness, hemophilia B, and lipoprotein lipase deficiency boosted scientists' interest in more carefully investigating a possible rAAV-based gene therapy approach for CF (Vidović et al., 2015). Here, rAAV is a derivate of wild-type $\mathrm{AAV}$ and is not related to human pathology; this vector holds promise because it is perceived to be a safe vector due to its low immunogenicity, lack of viral genes, and non-integrating character (Dismuke et al., 2014). Vidović et al. (2015) showed that the rAAV-mediated gene delivery of a shortened R domain deleted-CFTR led to the correction of the CF phenotype in $\mathrm{CF}$ mice nasal mucosa and in the intestinal organoids derived from CF patients. Scientists must now focus on methods to enlarge AAV tropism and to diminish its immunogenicity while improving CFTR expression and perseverance in the lungs. The efforts to improve the AAV vector efficacy in significant animal models of CF and the confirmation of the potent transduction of human epithelia for therapeutic use still remain challenging, but not unreachable (Hart and Harrison, 2017).

Another way to tackle packaging restraints and expand AAV tropism is the use of human Bocavirus-Type-1 (HBoV1) with AAV2 genome. Like AAV, the HBoV1 is a parvovirus that exhibits a high level of tropism for both transduced human cells polarized in the air-liquid interface cultures and for the apical membrane of the human airway epithelium. HBoV1 possesses a bigger capsid, allowing for the packing capacity of $5543 \mathrm{nt}$ compared with an AAV of 4679 nt. The chosen animal model for exploring therapeutic approaches with $\mathrm{rAAV} 2 / \mathrm{HBoV} 1$ vector has been $\mathrm{CF}$ ferrets because these animals mimic very well the physiological aspects of CF lung disease. The study by Yan et al. (2017) confirmed that in vitro and in vivo ferret epithelium is susceptible to transduction by the $\mathrm{rAAV} 2 / \mathrm{HBoV} 1$ vector. Moreover, the experiments showed that repetitive dosing in vivo was efficient in sustaining transgene expression (Yan et al., 2017).

\section{Non-viral Vectors}

The anxiety concerning unwanted immunogenic reactions, possible transgene miss-insertions, difficulty in packing a nucleic acid of an excessive size, and issues in bulk-production have shed light on non-viral vector alternatives (Foldvari et al.,
2016). Advancements in liposomal vectors have demonstrated the secured and feasible delivery of bulky DNA molecules (Foldvari et al., 2016; Hart and Harrison, 2017). A randomized, double-blind, placebo-controlled Phase $2 \mathrm{~b}$ trial carried out in the United Kingdom with a repeated nebulization of non-viral CFTR gene showed a modest improvement in $\mathrm{FEV}_{1}$ (forced expiratory volume in $1 \mathrm{~s}$ ) compared with placebo at 1 year, demonstrating the stabilization of the lung function in treated patients (Alton et al., 2015). The conclusion is speculative because the difference was mainly a consequence of decrease in the placebo group. Moreover, there was no evidence of WT-CFTR expression in respiratory cells (Mottais et al., 2017). Although disappointing, this trial demonstrated that dosing repetition is harmless, so there is a necessity to improve nucleic acid delivery to the target cell (Hart and Harrison, 2017).

To protect the DNA from extracellular factors such as mucus, bacteria, or inflammation and physical deterioration during inhalation, DNA nanoparticles can be shielded by biodegradable poly(b-amino esters) (PBAEs) polymers with a thick sheet of polyethylene glycol (Mastorakos et al., 2015). Furthermore, a cationic lipid labeled GL67A38 has shown endosomal discharge of plasmid DNA and stabilization during aerosol implementation although transfection efficiency was low (Dhand, 2017). Recent preclinical work has also demonstrated the ability of transferring circular pieces of DNA that retain transgene and regulatory elements by nanoparticles (Hart and Harrison, 2017). The use of synthetic vectors may also be considered when trying to decrease immunogenicity and improve integration (Lentz et al., 2005; Catanese et al., 2012).

\section{CRISPR/Cas9 Approach}

The CRISPR (clustered regularly interspaced short palindromic repeats)/Cas9 approach is a gene-editing strategy in which the specific mutated sequence of the defective CFTR gene is corrected by changes introduced into the DNA (Figure 3). CRISPR/Cas9 technology has been developed based on the bacterial defense mechanisms against "foreign" DNA (e.g., virus) (Alapati and Morrisey, 2017). In this mechanism, "foreign" DNA incorporates multiple small pieces into a locus consisting of short palindromic repeats, called CRISPR. Upon re-exposure to the introduced DNA, the CRISPR locus is transcribed into small RNAs that lead the Cas9 endonuclease to a particular spot in the added DNA, based on the DNA-RNA sequence complementarity, which creates a double-stranded opening and protects the host bacterium. CRISPR/Cas9 technology uses a protein-RNA complex composed of an enzyme-Cas9 endonuclease bound to a guide RNA (gRNA) molecule. Engineered Cas9 (Type II bacterial endonuclease) cleaves the DNA in a sequencespecific mode defined by the gRNA component that recognizes through the complementarity the mutated sequence and creates a specific double-stranded break (Figure 3). The cell can then fill the excised portion with the correct gene sequence through homologous directed repair (HDR), which is the desirable plan of action but can fail occasionally as in the case of the non-homologous end joining (NHEJ), which results in insertions/deletions formation. CRISPR has been recognized as 


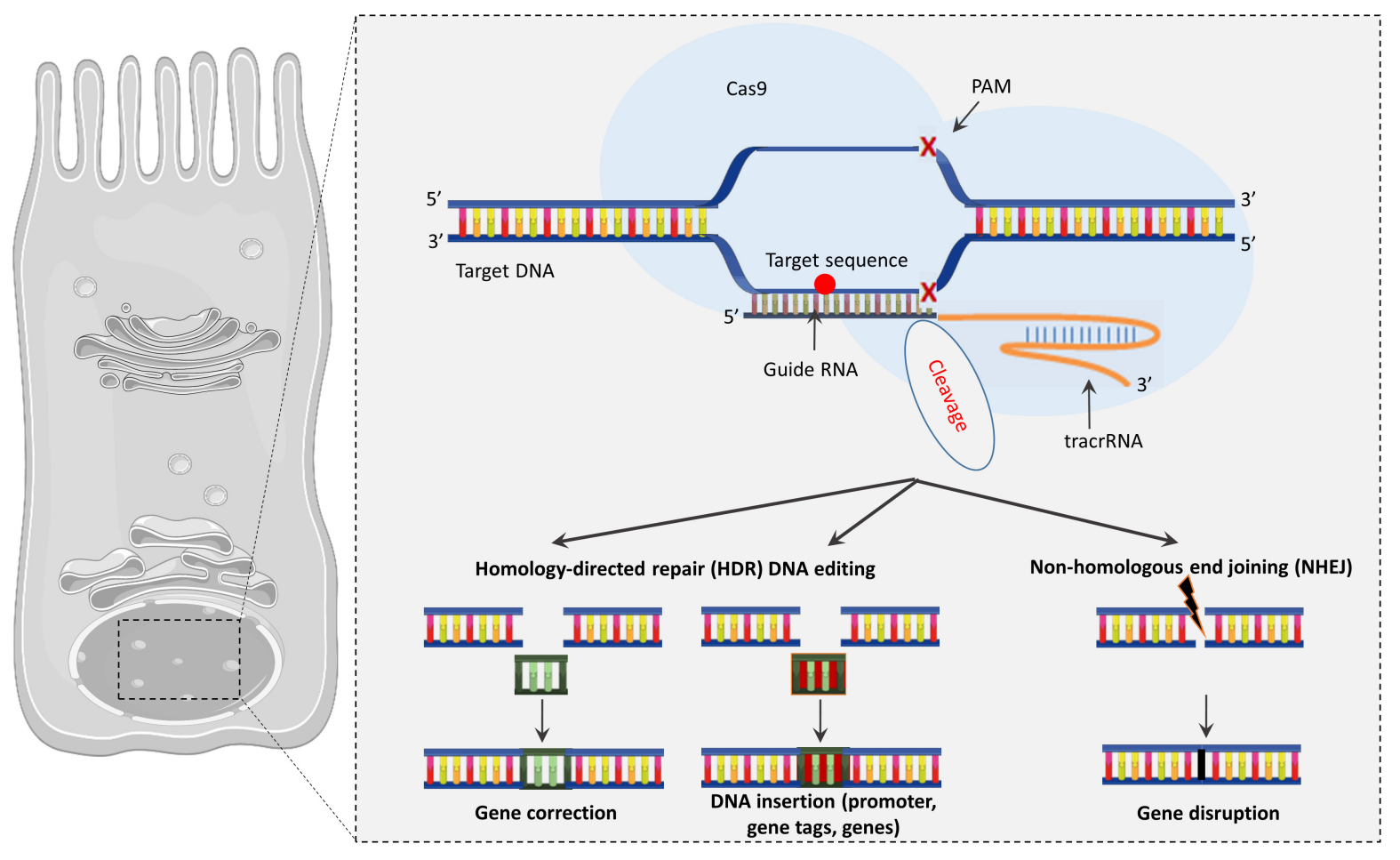

FIGURE 3 | The CRISPR/Cas9 system. Cas9 endonuclease binds to the target site using a guide RNA to precisely cut DNA allowing genome editing. CRISPR/Cas9 may drive to gene correction or DNA insertion through HDR or modify the sequence through NHEJ, which results in insertions/deletions.

the most powerful gene-editing tool when compared with zincfinger (ZFN) and transcription activator-like effector (TALEN) endonucleases. The designed gRNA has an identical sequence as the desired site in the genome, which allows for intervention at the level of the DNA sequence with high precision, acting with molecular "scissors" to cut the DNA at the desired point and replace it with the correct sequence (White et al., 2017).

The two main benefits of this precision-made correction of the faulty gene are as follows: (i) the modified gene prevails under control of its endogenous promoter, allowing life-long expression and natural adjustment in the cell; (ii) gene repair has the ability to bypass the engagement of the external DNA, hence decreasing the chance of insertional mutagenesis.

Non-viral (lipidic or polymeric) vectors seem suitable for achieving CRISPR/Cas9 expression probable transgene integration or secondary tumor initiation (Li et al., 2015). These vectors also prevent immune responses, which has been observed in the case of viral vectors, and limit off-target activity. The optimal mode of CRISPR/Cas9 introduction into the lungs is aerosol delivery devices combined with nanoparticle suspensions. Nevertheless, inhaled therapy can become entrapped and unable to cross the dense and viscous pathological mucus layer. Despite the fact that the CRISPR approach is in its early beginnings, it presents possible significant outcomes for future CF therapy benefits.

The first studies on CRISPR/Cas9 to develop a potential therapy for CF were published in 2013. Schwank et al. (2013) tested the recovery of a functional CFTR protein in intestinal organoids obtained from CF pediatric patients carrying the F508del mutation. After lipofectamine-mediated transfection of intestinal stem cells, CRISPR/Cas9 gene editing repaired the mutation at the CFTR locus through the CFTR gene substitution approach (HDR), improving the forskolin-induced response and prompting organoid swelling. The same group suggested colonic transplantation of genetically corrected organoids as a probable perspective (Yui et al., 2012). Another successful delivery of CRISPR/Cas9 was reported by Bellec et al. (2015). They knockeddown the CFTR gene in HAE cells and Calu-3 cells using the CRISPR/Cas9 approach delivered with HIV-1 lentivirus. CRISPR/Cas9-directed gene modification was associated with a decline of transepithelial $\mathrm{Cl}^{-}$secretion and a decrease in response to a CFTR inhibitor, as measured in polarized cell cultures in Ussing chambers.

Two other groups showed gene correction in CF-specific iPSCs (induced pluripotent stem cells) with the use of gene editing (Firth et al., 2015; Crane et al., 2015). Firth et al. (2015) used CRISPR technology and a piggyBac-based donor to obtain footprint-free gene correction at the CFTR locus in patient-derived iPSCs. The recovery of expression and function of CFTR, as measured by patch-clamp, in lung epithelial cells that were differentiated from edited iPSCs was demonstrated (Firth et al., 2015). Crane et al. (2015) used ZFN technology, which is less efficient than CRISPR, to correct the CFTR gene. When the repaired CF-iPSCs were differentiated into lung epithelial cells, a mature CFTR glycoprotein was expressed, which in turn recovered CFTR $\mathrm{Cl}^{-}$channel activity (Crane et al., 2015). 
Sanz et al. (2017) investigated the application of a CRISPR/Cas9-based NHEJ method to edit a small number of CF-causing mutations: c.1679 + 1634A > G, c.3140$26 \mathrm{~A}>\mathrm{G}$, and c.3718-2477C > T CF, which create alternative splice sites that produce pseudo-exons or extend existing exons. The group demonstrated that CRISPR Cas9/gRNA pairs are useful for successful excision via a NHEJ pathway. NHEJ-mediated excision took place in $\geq 25 \%$ of transfected cells, a degree of editing that is 10 -fold higher than their previous study of homology directed repair gene editing, with Cas9/gRNA in the same locus in the same cells (Hollywood et al., 2016).

\section{Antisense-Oligonucleotide-Mediated Therapy}

Antisense oligonucleotides (ASOs) are single-stranded synthetic RNA-like molecules that can selectively change gene expression by means of various techniques regulated by their chemistry and antisense oligomer design. Because these mechanisms of action are based on the complementary base pairing to the target sequences, oligonucleotides are highly specific compounds. Antisense oligonucleotides are being chemically modified to improve cellular uptake and intracellular stability and to decrease cell toxicity. From a pharmacological point of view, they are attractive forms of medication because they are resistant to nucleases and have good pharmacokinetic properties.

Zamecnik et al. (2004) constructed a modified oligodeoxyribonucleotide to replace the three missing bases caused by F508del mutation in the CFTR mRNA. This treatment in vitro showed improved CFTR function (Zamecnik et al., 2004). The company ProQR employs single-stranded antisense RNA-based oligonucleotides that encompass the lacking bases and behave as guide sequences to restore the targeted abnormal mRNA in cells carrying the F508del mutation. Phase II clinical trials with this modified RNA oligonucleotide QR-010 (Eluforsen), which is dedicated to F508del mutation, showed increased CFTR function in patients' nasal mucosa, good drug tolerance, and improved quality of life.

Antisense oligonucleotides can repair abnormal mRNA or alternatively target an RNA transcript for degradation through RNaseH activation. Recently, Crosby et al. (2017) tested ENaC-specific antisense oligonucleotides delivered by inhalation in mouse models for the prevention and reversal of lung symptoms in CF. Aerosol-delivered ENaC ASOs downregulated $\mathrm{ENaC}$ and mucus marker expression, ameliorated goblet cell metaplasia, inflammation status, and airway hyperresponsiveness (Crosby et al., 2017).

Most of the efforts in antisense oligonucleotides research focus on splicing alteration to skip an exon enclosing a nonsense or frameshift mutation or alternatively recover the reading frame, expecting here that the end isoform will retain improved function compared with the mutated protein. ASOs have also been shown to modulate splicing in cells with the CFTR splicing mutation c. $2657+5 \mathrm{G}>\mathrm{A}$, which causes exon 16 to be omitted along the splicing (Igreja et al., 2016). Single-stranded DNA oligonucleotides of 19 nucleotides having 2'-O-methyl modified ribose and a phosphorothioate backbone were modeled to hybridize to pre-mRNA and correct aberrant splicing in HEK293 cells expressing the c. $2657+5 \mathrm{G}>\mathrm{A}$ mutant CFTR minigene (Igreja et al., 2016). A similar correction was achieved for $3849+10 \mathrm{~kb} \mathrm{C} \rightarrow \mathrm{T}$, a mutation identified in $5 \%$ of Ashkenazi Jewish patients, which constitutes a novel donor site in intron 19, causing an 84 base-pair pseudo-exon to be incorporated into the mRNA, and generating a downstream PTC (Friedman et al., 1999).

\section{mRNA-Mediated Therapy}

Whereas conventional gene therapy targets the nucleus, in mRNA therapy, a right nucleotide sequence coding for CFTR is targeted at the cell cytoplasm. In both cases, a normal protein is produced, though the concepts based on mRNA delivery are more convincing because they are not required to overcome the nuclear membrane barrier. Apart from this, chemically changed mRNA displays various benefits over other nucleic acids. The most valuable characteristics are a transient protein expression, decreased immunogenicity, superior translation efficacy, enhanced stability, and pharmaceutical safety because modified mRNA is not incorporated into the host genome (Kuhn et al., 2012). The mRNA can be delivered to the cell by using liposomal or polymeric non-viral vector formulations that are administered via several routes, for example, intraperitoneally, intravenously, or intratracheally.

The transfection of chemically modified WT-CFTR mRNA in CFBE41o-cells homozygous of the F508del mutation reestablished cAMP-induced CFTR currents (measured in Ussing chambers) similar to WT cells as a result of the mRNAdriven replacement of functional channels (Bangel-Ruland et al., 2013). Immunofluorescence and biochemical approaches have confirmed the expression of apically located WT-CFTR after optimized WT-CFTR mRNA transfection. Primary cultured human nasal epithelial cells were characterized by nearly a twofold improvement in the cAMP-stimulated CFTR current after mRNA transfection.

Robinson et al. (2018) used a clinically relevant lipid-based nanoparticle (LNP) for the packaging and transport of large chemically modified CFTR mRNA (cmCFTR) to bronchial epithelial cells derived from patients. The experiments showed an increase in membrane-localized CFTR and rescue of its primary function as a $\mathrm{Cl}^{-}$channel. Nasal application of LNP-cmCFTR recovered CFTR-mediated $\mathrm{Cl}^{-}$secretion to conductive airway epithelia in CFTR knockout mice for at least 14 days. The CFTR activity peaked on the 3 rd day post-transfection, retrieving up to $55 \%$ of the net $\mathrm{Cl}^{-}$efflux that is distinctive for the healthy mice (Robinson et al., 2018).

\section{STEM CELL THERAPY}

The biggest obstacle to overcome in regenerative medicine is to determine the relevant cells that will be capable of repairing a defect. The desired cell should be non-immunogenic, patient specific, easy to make proliferate, and easy to modify (Guo et al., 2017). Because endogenous progenitor cells are difficult to 
recognize and insufficient in their quantity and activity, focus has been directed on exogenous cell sources (McQualter et al., 2010; Rock and Königshoff, 2012). The discovery of iPSCs inspired a discussion about whether iPS cells are a copy of embryonic stem cells (ESCs). Yamanaka's group first obtained the iPS cells by reprogramming mouse fibroblasts and next confirmed the results with the use of human fibroblasts (Takahashi et al., 2007). Nowadays, the existing therapeutic probability of ESCs is even more alluring when combined with the CIRSPR/Cas9 approach (Wagner et al., 2016). Indeed, repairing a CFTR mutation has been demonstrated in human iPSC cells by means of CRISPR/Cas9. Skin fibroblasts derived from CF individuals were reprogrammed toward iPSCs, transfected with the CFTR/Cas9 gRNA vector, corrected to their WT phenotype, and further entirely differentiated into cells of proximal and distal airways (Crane et al., 2015; Pollard and Pollard, 2018). This suggests that theoretically, stem lung cells restored to their WT phenotype could be regrafted to lung niches to further redifferentiate them into respiratory cells.

Moreover, the CRISPR/Cas9 method showed the total recovery of CFTR protein function in organoids-intestinal stem cells placed in culture-derived from children with CF (Schwank et al., 2013). The possibility to engraft cultured colon organoids was tested in a mouse by using effective transplantation into a superficially damaged mouse colon. The integration of grafted cells into the mouse colon resulted in a coating with epithelium in the deprived area of the damaged colon (Yui et al., 2012; Firth et al., 2015). However, this technology is time- and labor-intensive and challenging in terms of obtaining entirely differentiated lung-specific cell subsets (Wagner et al., 2016).

Presumably, ESCs/iPSCs assays will become a potent method to better forecast patients' clinical responses to CFTR modulators. The creation of an array of iPS cell lineages possessing the characteristics of different CF mutations will provide a powerful tool for selecting the potential drug to repair functional deficiencies (Schmidt et al., 2016; Simsek et al., 2016; Conese et al., 2018). Importantly, safety concerns about ESC- and iPSCbased cell grafts, including the transmission of possible genetic abnormalities and tumor risk, may hamper potential clinical therapeutic purpose. A clear answer is needed to define if the mutations were already pre-existing in the cells of origin or if they were introduced during the reprogramming process. Future preclinical risk assessments need to better establish tumor and disease risk related to the therapeutic use of iPSC derivatives (Martin, 2017).

\section{READ-THROUGH THERAPIES AND NMD INHIBITORS}

Read-through agents (Table 2) are directed at in-frame nonsense mutations. Some PTCs are more "permissive" than others to NMD, leading to residual levels of mRNA. This residual level of mRNA is the target for "read-through" agents, whose goal is to reduce the ribosomal ability to proofread and enable ribosomes to skip the PTC, leading to the formation of functional protein. Several factors are challenging when it comes to read-through treatment efficacy: (i) the level of drug-induced read-through, (ii) the amount of target transcripts, and (iii) the activity of the recoded protein (Pranke et al., 2018).

Aminoglycosides were the first read-through agents tested for CF disease (Howard et al., 1996). Aminoglycoside antibiotics are amino sugars that interact with the ribosome at the A-site and imitate a conformational change in the ribosomal RNA that normally is induced by codon-anticodon pairing; therefore, this promotes near-cognate tRNA incorporation and increases the number of PTC misreading, allowing translation to continue to the correct termination codon. Gentamicin and geneticin present read-through potential (Wilschanski et al., 2000; Wilschanski et al., 2003; Kandasamy et al., 2012). Gentamicin was found to display beneficial effects in patients with at least one Class I mutation, as assessed by an improvement in nasal potential difference after topical nasal application (Wilschanski et al., 2003) and intravenous administration (Sermet-Gaudelus et al., 2007). These small-scale clinical trials provided a proof-of-concept for read-through efficiency. However, gentamicin and geneticin cannot be used in clinics because of serious renal toxicity and ototoxicity. Several studies have been performed to develop chemically modified aminoglycosides to provide higher activity and less toxicity (Nudelman et al., 2009; Rowe et al., 2011; Xue et al., 2014). NB124 (ELX-02, ELOXX Pharmaceuticals) is a derivative of aminoglycoside that is modified to provide a higher level of read-through activity than gentamicin (Xue et al., 2014). It has been shown that NB124 restores CFTR function to roughly $7 \%$ of WT levels. Its read-through potency has been shown in respiratory cell lines for the most prevalent PTCsG542X, R553X, R1162X, and W1282X-and in primary human bronchial epithelial (HBE) cells from patients carrying the G542X mutation. Moreover, tests of ototoxicity in the tissue-based model showed that this compound is also less cytotoxic than gentamicin. Lower levels of toxicity and a higher level of PTC suppression by NB124 are achieved through a strong preference for cytosolic versus mitochondrial ribosomes. A Phase II clinical trial will begin in 2019 in Belgium.

High-throughput screening (HTS) identified PTC124 (Ataluren $^{\circledR}$, Translarna ${ }^{\mathrm{TM}}$ PTC Therapeutics) (Welch et al., 2007). The systemic administration of PTC124 in CF mice expressing a human CFTR-G542X transgene induced CFTR function rescue. This small molecule was tested in a Phase II clinical study (Kerem et al., 2008; Sermet-Gaudelus et al., 2010) with contradictory results. A first Phase III trial did not deliver any significant changes in $\mathrm{FEV}_{1}$ although there was a positive trend favoring Ataluren ${ }^{\circledR}(-2.5 \%$ change $)$ over the placebo $(-5.5 \%$ change; $p=0.124)$ (Wilschanski et al., 2011). New clinical placebo-controlled Phase III trials excluding inhaled tobramycin, which could antagonize the effect that PTC124 has on the ribosome, demonstrated no evidence in improving $\mathrm{FEV}_{1}$, nor any benefit in bronchial exacerbations. The clinical development of the compound is now stopped in the field of CF (Kerem et al., 2014).

Other factors may also influence the read-through efficiency, for example the PTC identity, the neighboring mRNA sequence, the NMD efficiency controlling the level of mRNA, the geometry of the tRNA-mRNA complex in the presence of the drug at 
the ribosome-decoding center, and the function of the protein, which is neoformed.

Therefore, new tracks of research for PTC-associated mutations are now being explored, including (i) NMD inhibitors, (ii) the association with CFTR modulators to improve CFTR expression/function of the neoformed protein, and (iii) factors that force mismatched base pairs to adopt a Watson-Crick geometry.

ReCode Therapeutics has been developed as an innovative therapeutic approach that utilizes suppressor transfer RNA (tRNAs) with the goal of correcting CF-causing nonsense mutations. RCT101 is a therapeutic nucleic acid actively studied in preclinical models. This modified tRNA is transported to cells by patented nanoparticles to correctly "recode" the translating protein. The unpublished data from experiments on human HBEs from patients with genotypes G542X/G542X and F508del/G542X showed that RCT101 significantly increased CFTR-dependent $\mathrm{Cl}^{-}$secretion as a single active agent or in combination with VX-809 and VX-770 (Hagemeijer et al., 2018).

\section{CFTR MODULATOR THERAPIES}

A CFTR modulator is a pharmaceutical agent that targets a specific defect in the CFTR protein that is caused by mutation in the CFTR gene. This modulator does not correct mutations in the gene but rather targets the errors that occur posttranscriptionally, either during protein folding, trafficking up to the PM, or CFTR functioning.

The CFTR modulators are classified into four main groups: potentiators, correctors, amplifiers, and stabilizers (Table 2).
They are different in their mechanisms of action, which can be determined by the type of protein defect they target (Sloane and Rowe, 2010).

\section{Potentiators}

Therapeutic agents that improve the channel-open probability and potentiate mutated CFTR gating are called potentiators. A large number of proof-of-concept studies have been published to demonstrate that ATP analogs and small-molecule agents have good potentiation activity. Chemically modified analogs of ATP have demonstrated a significant increase in the open probability of CFTR, with P-ATP [ $N^{6}-(2$-phenylethyl)-ATP] having the highest affinity and efficacy (Zhou et al., 2005; Bompadre et al., 2008). It has been demonstrated that P-ATP increases the open probability of G551D-CFTR and F508del-CFTR (Miki et al., 2010). Another ATP analog 2'-dATP (2'-deoxy-ATP) also enhances the gating of WT-CFTR and G551D-CFTR (Aleksandrov et al., 2002; Cai et al., 2006). Remarkably, both modifications in ATP have synergic effects in the potentiating gating of G551D- and F508del-CFTR (Miki et al., 2010). Nevertheless, issues with bioavailability and potential unspecific binding to other proteins involved in multiple physiological functions limit the utility of ATP analogs in clinics.

The agents that increase the intracellular concentration of cAMP and hence amplify PKA activity and the phosphorylation level of the R domain might also improve the activity of defective CFTR. First, Drumm et al. (1991) found that IBMX (3-isobutyl-1methylxanthine), a compound that increases intracellular cAMP by inhibiting phosphodiesterase, increases PKA activity and makes F508del-CFTR more responsive to activation. Second, an isoflavone derivative genistein is a tyrosine kinase inhibitor that

TABLE 2 | Strategies of treatment for personalized CF medication and compounds tested pre-clinically and clinically.

\begin{tabular}{|c|c|c|}
\hline \multicolumn{2}{|c|}{ Therapy } & \multirow{2}{*}{$\begin{array}{l}\text { Compounds } \\
\text { Geneticin, RCT101 }\end{array}$} \\
\hline Read-through agents & Pre-clinical & \\
\hline & Clinical & Gentamicin $\downarrow$, NB124 (ongoing), PTC124 $\downarrow$ \\
\hline \multirow{2}{*}{ Potentiators } & Pre-clinical & $\begin{array}{l}\text { ATP analogs (P-ATP, 2'-dATP, P-dATP), IBMX, PG-01, VRT-532, dihydropyridine } \\
\text { blockers of L-type calcium channels, CO-068, CB subunit of crotoxin, P5, G01, } \\
\text { A01, A02, H01, H02, H03 }\end{array}$ \\
\hline & Clinical & $\begin{array}{l}\text { Genistein } \downarrow \text {, curcumin } \downarrow, \text { VX-770 }, \text { PTI-808 (ongoing in triple combination), } \\
\text { GLPG1837 (ongoing) }\end{array}$ \\
\hline \multirow{2}{*}{ Correctors } & Pre-clinical & $\begin{array}{l}\text { Curcumin, HDAC inhibitors (SAHA), Corr-4a, VRT-325, glafenine, RDR1, } \\
\text { 407882, FDL169, }\end{array}$ \\
\hline & Clinical & $\begin{array}{l}\text { 4PBA } \downarrow \text {, miglustat } \downarrow \text {, sildenafil } \downarrow, \text { VX-809 (Orkambi }{ }^{\circledR} \text { ) } \uparrow, \text { VX-661 (Symdeko }{ }^{\circledR} \text { ) } \uparrow \text {, } \\
\text { VX-440, VX-152, VX-659, VX-445 (ongoing in triple combinations), cavosonstat } \\
\text { (ongoing), GLPG2222, GLPG2851, GLPG2737, GLPG3221 (ongoing in triple } \\
\text { combinations), PTI-801 (ongoing in triple combination) }\end{array}$ \\
\hline Amplifiers & Clinical & PTI-428 (ongoing in triple combination) \\
\hline \multirow{2}{*}{ Stabilizers } & Pre-clinical & HGF (hepatocytes growth factor), VIP (vasoactive intestinal peptide) \\
\hline & Clinical & Cavosonstat \\
\hline
\end{tabular}

$\uparrow$-indicates succesfull clinical outcome and the FDA approval, $\downarrow$-indicates unsuccesfull clinical attempt. 
can also activate CFTR in intact cells (Illek et al., 1995, 1996). Illek et al., (1995 1996) proposed that this effect was mediated by increasing intracellular cAMP; however, other independent studies have demonstrated that genistein directly targets and binds to the CFTR protein (French et al., 1997; Hwang et al., 1997; Weinreich et al., 1997). Genistein entered the clinical study as a duo therapy with phenylbutyrate; however, the results were not satisfying. Other flavonoids (apigenin, kaempferol, and quercetin) have been found to enhance the currents in vitro and in vivo by increasing the CFTR channel's open probability (Illek and Fischer, 1998). Naturally existing curcumin also extends the channel opening duration of WT-CFTR (Berger et al., 2005), F508del-CFTR (Berger et al., 2005), and G551D-CFTR (Wang et al., 2007) although data from clinical investigations have not been conclusive. Similarly, it was proposed that sildenafil, a phosphodiesterase (PDE5) inhibitor, activates the guanylate cyclase and increases the intracellular cGMP and hence CFTR activity (Lubamba et al., 2008).

First cell-based fluorescence HTSs by Verkman's laboratory (Yang et al., 2003; Pedemonte et al., 2005b) and Vertex Pharmaceuticals (Van Goor et al., 2006), applying a Fischer rat thyroid (FRT) cell line stably expressing F508del-CFTR and an YFP sensitive to iodide, led to the discovery of several classes of small-molecule potentiators (Yang et al., 2003). Screening realized by Pedemonte et al. (2005b) showed the ability of the phenylglycine molecule PG-01 to restore the opening of F508del-CFTR almost to the level of WT-CFTR. Van Goor et al. (2006) identified VRT-532 (pyrazole). As shown by Pasyk et al. (2009) and Wellhauser et al. (2009), VRT-532 potentiates the gating of G551D- or F508del-CFTR through direct interaction with CFTR and the restoration of its defective ATPase activity (Pyle et al., 2011).

In 2009, Vertex Pharmaceuticals discovered VX-770 (Ivacaftor), which potentiates CFTR activity. First, they showed that VX-770 increases the activity of F508del- and G551D-CFTR using the patch-clamp technique and $\mathrm{Cl}^{-}$secretion measures in bronchial epithelial cell cultures sampled from patients carrying these mutations (Goor et al., 2009). VX-770 prolongs the opening duration of WT-CFTR (Hwang and Sheppard, 2009), acting independently of ATP hydrolysis and NBD domain dimerization, because VX-770 efficiently potentiates G551D-CFTR (Van Goor et al., 2009; Eckford et al., 2012; Jih and Hwang, 2013), E1371SCFTR (Jih and Hwang, 2013), and WT-CFTR in the absence of ATP (Eckford et al., 2012; Jih and Hwang, 2013). Eckford et al. (2012) suggested that VX-770 interacts directly with CFTR and induces an unconventional mode of gating. Although VX-770 increased the residual forskolin-stimulated channel activity in HBE cell cultures from some F508del-homozygous patients (Van Goor et al., 2009), a Phase II investigation of F508del-homozygous patients showed no improvement in $\mathrm{FEV}_{1}$ (Flume et al., 2012).

A Phase II and Phase III study in patients carrying the G551D mutation showed that ivacaftor efficiently improved predicted $\mathrm{FEV}_{1}$ in as early as 15 days, reaching a $10.6 \%$ increase in $\mathrm{FEV}_{1}$ at 24 weeks of treatment $(p<0.001)$. Ivacaftor decreased sweat chloride concentration by $48 \mathrm{mmol} / \mathrm{l}$ compared with the placebo $(p<0.001)$, reduced the frequency of pulmonary exacerbations by $55 \%(p=0.001)$, and increased the weight of patients to $2.7 \mathrm{~kg}(p<0.001)$ (Ramsey et al., 2011). Phase II trials also showed significant improvements of the channel function in the nasal and sweat gland epithelia (within-subject) (Accurso et al., 2010). Children with "silent lung disease," which is characterized by normal initial $\mathrm{FEV}_{1}$, also demonstrated a significant improvement in $\mathrm{FEV}_{1}$ and lung clearance index. These results demonstrated that correcting CFTR at the molecular level can translate into outstanding clinical improvements. VX770 (trade name Kalydeco ${ }^{\circledR}$ ) became the first CFTR modulator approved for use in clinics and was initially approved in the United States (beginning of 2012), then in Europe and Canada (end of 2012), Australia, and New Zealand (2013).

Further development of ivacaftor demonstrated its clinical benefit in eight additional Class III gating mutations, including S549N and G551S, confirming improvement in lung function, BMI, sweat chloride, and CFQ-R; in addition, this method did not have safety concerns (De Boeck et al., 2014). Ivacaftor proved effective in a preschool population in open-label studies, highlighting an increase in fecal elastase and potential reversal in early pancreatic insufficiency status. An important concern was abnormalities of liver function tests in this population. Finally, ivacaftor demonstrated substantial activity in the non-gating mutation $\mathrm{R} 117 \mathrm{H}$, with amelioration in sweat chloride and CFQ$\mathrm{R}$ scores in all age groups, whereas respiratory improvement was significant only in adults, perhaps because of the disease being more established in these patients (Yu et al., 2012; Carter et al., 2015).

Given these outstanding clinical benefits, the Food and Drug Administration (FDA) approved ivacaftor for marketing authorization based on in vitro assays for a number of mutations with residual function. Nowadays, ivacaftor is approved by the FDA for patients aged 1 and older who have one of the following gating mutations: G178R, S549N, S549R, G551D, G551S, G1244E, S1251N, S1255P, or G1349D; one of the following residual function mutations: A455E, E193K, R117C, A1067T, F1052V，R347H，D110E，D110H，F1074L，R352Q，G1069R, R1070Q, D579G, K1060T, R1070W, D1152H, L206W, S945L, D1270N, P67L, S977F, E56K, or R74W; one of the following splice mutations: $711+3 \mathrm{~A} \rightarrow \mathrm{G}, 3272-26 \mathrm{~A} \rightarrow \mathrm{G}, \mathrm{E} 831 \mathrm{X}, 2789+5 \mathrm{G} \rightarrow \mathrm{A}$, or $3849+10 \mathrm{~kb} \mathrm{C} \rightarrow \mathrm{T}$; or the conduction mutation $\mathrm{R} 117 \mathrm{H}$.

Since the approval of ivacaftor, many other potentiators have been found. A number of these are still in preclinical development. A screen of the approved drugs performed by Galietta's laboratory picked out dihydropyridine blockers of L-type calcium channels to have the potentiation activity of F508del-CFTR (Budriesi et al., 2011); however, their clinical usefulness is unclear because of side effects (e.g., off-target cardiac effect). In turn, Faure et al. (2016) showed that a CB subunit of crotoxin from Crotalus durissus terrificus interacts with the NBD1 domain of both WT- and F508del-CFTR and increases their $\mathrm{Cl}^{-}$channel currents. To identify the potentiators that act synergistically with correctors, Verkman's laboratory screened the analogs of previously found P5 potentiators and unrelated synthetic small molecules. They found 12 of the most active compounds, including a thiophene (G01), a 2-thioxo-4amino-thiazoles (A01 and A02), and pyrazole-pyrrole isoxazoles 
( $\mathrm{H} 01, \mathrm{H} 02$, and H03), with a higher potentiating efficacy in FRT cells than VX-770.

An investigational CFTR potentiator proposed by Proteostasis Therapeutics-PTI-808-was found to enhance the function of F508del-CFTR (2018 ECFS Conference, New Frontiers in Basic Science of Cystic Fibrosis) and is currently in Phase I clinical trials together with PTI-801 and PTI-428 as a combination therapy.

Interestingly, dihydro-5H-thieno[2,3-c]pyran-2-yl)-1Hpyrazole-3-carboxamide) GLPG1837, a more recent potentiator developed by Galapagos, exhibits a higher efficacy than VX770 for G551D-CFTR (Van der Plas et al., 2018). Similar to VX-770, GLPG1837's underlying mechanism is independent of NBD domain dimerization and ATP hydrolysis. By applying GLPG1837 with VX-770 together, Yeh et al. (2017) provided evidence that these two molecules probably act in competition for the same site of action, whereas GLPG1837 and the ATP analog P-dATP work synergistically through two different sites. Two Phase II clinical studies are now conducting enrollment to test the GLPG1837 compound in patients with a S1251N mutation and G551D mutation.

\section{Correctors}

The CFTR correctors are small molecules that improve the trafficking of mutated CFTR (Class II mutations, e.g., F508del) from ER to the apical PM and increase CFTR cell surface expression. These correctors improve defective CFTR folding and cellular processing by direct binding (called pharmacological chaperones) or modulate protein homeostasis and the quality control system of the cell to modify the recognition and processing of misfolded CFTR (called proteostasis regulators). Because F508del-CFTR presents multiple defects, the development of correctors is a greater challenge than the development of potentiators (Thibodeau et al., 2010). Indeed, correction of F508del-CFTR requires the following: (i) rescue to native folding by the restoration of NBD1 energetics and interface instability; (ii) evasion of the protein from ER quality control; (iii) enhancement in the apical cell membrane localization; and (iv) improvement in CFTRdependent $\mathrm{Cl}^{-}$secretion. Hence, a strategy to combine the correctors with potentiators and even an amplifier or stabilizer into a "combination therapy" was tested in vitro and in clinical trials.

The initial studies focused on the regulation of proteostasis for F508del-CFTR. Early studies have shown that some drugs approved for other diseases have the corrector activity for F508del-CFTR. The compound 4-phenylbutryate (Buphenyl, $4 \mathrm{PBA}$ ), a chemical chaperone that stabilizes the folding of proteins, has been found to increase F508del-CFTR PM expression in cell culture models (Rubenstein and Zeitlin, 2000). However, this clinical trial failed to confirm the correction activity, as measured by nasal potential difference (Zeitlin et al., 2002). Curcumin (Egan et al., 2004; Lipecka et al., 2006) blocks calcium from entering into the ER and thus may interfere with the calcium-dependent chaperones that are involved in the degradation of the CFTR. Initial tests in patients failed to confirm any efficacy. Miglustat (n-butyldeoxynojyrimicin) is an alpha-glucosidase inhibitor that may interfere with F508delCFTR misfolding quality control (Noël et al., 2008; Robert et al., 2008; Norez et al., 2009). Although Miglustat efficiently corrected the functional cell surface expression of F508delCFTR in cell culture models and mice, a small clinical study did not confirm the corrector activity. Wang et al. (2006) demonstrated that increased PM localization of F508del-CFTR can be achieved by the down-regulation of Ahal (an Hsp90 cochaperone), whereas Hutt et al. (2010) proposed that the inhibition of histone deacetylase HDAC7 activity with HDAC inhibitors (Suberoylanilide Hydroxamic Acid, SAHA) facilitates F508del-CFTR folding and stability and corrects F508del-CFTR. However, a later study by Bergougnoux et al. (2017) had contradictory conclusions, with experiments in human nasal epithelial (HNE) cells showing that SAHA decreased CFTR transcript and protein levels.

A second strategy was based on the HTSs of small molecules. The first corrector identified with this methodology was bithiazole Corr-4a (bisaminomethylbithiazole, C4) (Pedemonte et al., 2005a), whose later analogs have improved potency (Yu et al., 2008). Corr-4a stabilizes both ER- and PM-localized F508del-CFTR. This improves the domain assembly (Loo et al., 2008, 2009) rather than NBD1 stability (Farinha et al., 2013; Okiyoneda et al., 2013). HTSs by Vertex Pharmaceuticals introduced new correctors (Van Goor et al., 2006), including VRT-325 (quinazolinone, C3), which stabilizes both the ERand PM-localized F508del-CFTR by improving domain assembly (Loo et al., 2008, 2009). Both Corr-4a and VRT-325 may not function through direct binding to CFTR because they are not specific to CFTR (Van Goor et al., 2006, 2011). Although Corr-4a and VRT-325 present a correction activity in vitro, they did not find a pharmacological use because of the high toxic effects and low in vivo efficacy.

Other small-scale screenings have provided further correctors, such as the drug glafenine (Robert et al., 2010), phenylhydrazone RDR1 (Sampson et al., 2011), and candidate molecules from computational screening (Kalid et al., 2010). These compounds are relatively less efficacious and have not been tested in clinical studies. Finally, a structure-based virtual screening by Odolczyk et al. (2013) developed other small compounds that rescue F508del-CFTR functional cell surface expression by inhibiting the interaction of F508del-CFTR with keratin 8 (Colas et al., 2012).

Following HTS by Vertex Pharmaceuticals and chemical optimization, the most promising compound VX-809 (lumacaftor, VRT-826809) became the first corrector approved for clinical use as a combined oral treatment with VX-770 (trade name of combination Orkambi ${ }^{\circledR}$ ). VX-809 has a higher potency and efficacy than VRT-325 and Corr-4a (Van Goor et al., 2010, 2011; Farinha et al., 2015). In F508del-homozygous HBE cells, VX-809 rescued total $\mathrm{Cl}^{-}$secretion up to $\sim 14 \%$ of WT-HBE cells (Van Goor et al., 2011) and even up to $25 \%$, as evaluated in HBE/HNE cells (Pranke et al., 2017). The VX-809 correction effect on F508del-CFTR is additive to VRT-325 and Corr-4a, suggesting a different mode of action (Van Goor et al., 2011). Interestingly, the correction effect of VX-809 can also be unmasked in HAE cells carrying only one copy of the F508del 
mutation: F508del/D1152H, F508del/394delTT, F508del/1717$1 G$ > A (Pranke et al., 2017), F508del/G542X (Awatade et al., 2015; Pranke et al., 2017), F508del/Y1092X (Awatade et al., 2015), F508del/R117H, F508del/W1282X, or F508del/E60X (Gentzsch et al., 2016). Conversely, N1303K-CFTR, another Class II mutant, was not corrected by VX-809 in HBE cells, but R117H- (Gentzsch et al., 2016) and A561E-CFTR (Awatade et al., 2015) efficiently improved PM localization and function. The mechanism of action is not yet fully known; however, it has been shown that VX-809 stabilizes TMD1 (Loo et al., 2013; Okiyoneda et al., 2013), improves TMD1 folding (Ren et al., 2013), and stabilizes interdomain interactions between TMDs and NBDs (He et al., 2012; Loo and Clarke, 2017). Studies on in vitro liposomes and C18 analogs of VX-809 (Eckford et al., 2014) applying the alkyne-containing VX-809 derivatives (Sinha et al., 2015) have shown that VX-809 may bind to CFTR directly; however, the exact binding site has not been found. Evidence that VX-809 binds directly to CFTR is based on the precipitation of the VX-809-bound CFTR and on the visualization of VX809-CFTR association in cells, thanks to the fact that the alkyne group of VX-809 derivatives can be conjugated to biotin-azide molecules through $\mathrm{Cu}$ '-catalyzed cycloaddition and by applying the conjugated biotin moiety.

As a whole, F508del-CFTR correctors in a single compoundtreatment present modest effectiveness in CFTR rescue. It has been proposed that this limited efficacy is caused by the necessity to rescue multiple defects in F508del-CFTR at the same time (folding, activity, and stability defects) (Sloane and Rowe, 2010). This was illustrated by a clinical trial testing efficiency of VX809 alone in F508del homozygote patients, which failed to demonstrate any improvement (Clancy et al., 2012).

Considering the unsatisfying results of the VX-809 corrector, Vertex Pharmaceuticals proposed to combine the potentiator (VX-770) and corrector (VX-809) because the in vitro analysis demonstrated that ivacaftor increased the open probability of F508del-CFTR by fivefold. Acute administration of VX770 to VX-809-corrected primary HAE cells increased the F508del-CFTR function (Van Goor et al., 2011). However, later analysis of $\mathrm{HBE}$ cells showed a reduction in the correction efficacy of VX-809, as well as VX-661 when VX-770 was applied chronically (Cholon et al., 2014; Veit et al., 2014). Chronic co-treatment with VX-809 and VX-770 affected the folding efficiency of F508del-CFTR at the ER and its metabolic stability in Golgi apparatus and PM, reducing the F508del-CFTR density at the apical PM and function (Veit et al., 2014).

Initially, two Phase III clinical studies (TRAFFIC and TRANSPORT) were designed to assess the efficacy and safety of two different doses of VX-809 in combination with VX770 in F508del-homozygous patients (Wainwright et al., 2015). Phase III clinical trials were shown to provide a benefit for patients. Patients over 12 years of age treated with the VX809/VX-770 combination therapy for 24 weeks showed a mean absolute improvement in $\mathrm{FEV}_{1}$ between active treatment and placebo ranging from 2.6 to 4.0 percentage points $(P<0.001)$, a statistically significant weight gain, reduction in pulmonary exacerbations, and fewer hospitalizations. There were no major safety concerns. However, some increases in blood pressure and chest tightness/bronchospasm were reported (Wainwright et al., 2015). In 6-11-year-old patients, VX-770/VX-809 combination therapy demonstrated a statistically significant improvement in the lung clearance index (LCI) (-1.09 Unit; $p<0.0001), \mathrm{FEV}_{1}$ $(+2.4 \% ; p=0.02)$, sweat chloride $(-21 \mathrm{~mm} / \mathrm{l} ; p<0.0001)$ and body mass index $Z$-scores $(+0.15, p<0.0001)$ (Ratjen et al., 2017). Importantly, long-term follow up of patients on VX770/VX-809 now show a slower decline in lung function over the study period compared with the rate of decline anticipated from registry data of patients not on VX-770/VX-809 (Konstan et al., 2017). Although the improvements seen with VX-770/VX809 in F508del homozygotes were lower than those seen in VX-770 responsive mutations, the FDA and EMA approved this combination therapy (trade name Orkambi ${ }^{\circledR}$ ) in 2015 for patients ages 12 and older who have two copies of the F508del mutation. In 2016, the FDA extended the license to patients aged 6-11 years and in August 2018 patients 2 years of age and older. However, it must be pointed out that clinical studies with Orkambi ${ }^{\circledR}$ showed a variable clinical responsiveness among patients. Less than 50\% of patients had a $\mathrm{FEV}_{1}$ improvement by more than $5 \%$, and only $25 \%$ of patients improved by more than $10 \%$ (Wainwright et al., 2015). This issue underlies the importance of the preclinical evaluation of CFTR modulators for each patient with the use of patient-specific biomarkers predictive of clinical efficacy.

A subsequent corrector developed by Vertex Pharmaceuticals-VX-661 (Tezacaftor)-has a structure similar to VX-809 but optimized pharmacokinetic properties. As reported by Van Goor et al., VX-661 increases $\mathrm{Cl}^{-}$transport in F508del-homozygous HBE cells (from 2.5 to $8.1 \%$ of normal levels in WT-HBE) and in other additional CFTR mutant HBE cells (including mutations associated with gating defects and residual CFTR function) (Fidler et al., 2017). The combination of VX-661 and VX-770 increases $\mathrm{Cl}^{-}$transport to $15.7 \%$ of normal $\mathrm{Cl}^{-}$transport and improves ciliary beat frequency and fluid transport. Pranke et al. (2017) confirmed these results in $\mathrm{HBE} / \mathrm{HNE}$ cells from F508del-homozygous patients and showed that $\mathrm{Cl}^{-}$secretion increased up to $27.4 \%$ of normal WT cells. An increase of $\mathrm{Cl}^{-}$transport was also measured in HBE cell cultures from heterozygous patients with genotypes F508del/394delTT and F508del/1717-1G > A (Pranke et al., 2017). After successful preclinical tests of VX-661, Vertex Pharmaceuticals next proposed a combination therapy of VX-661 and VX-770 originally for patients with two copies of the F508del mutation. Two separate multicenter clinical studies assessed the efficacy and safety of VX-661/VX-770 in patients 12 years of age and older: the EVOLVE study (Phase 3, randomized, double-blind, placebo-controlled, parallel group study) for patients homozygous for the F508del mutation; and the EXPAND study (Phase 3, randomized, double-blind, placebo-controlled, crossover study) for patients with one mutation that results in residual CFTR function in trans with the F508del mutation. The mean absolute improvement in $\mathrm{FEV}_{1}$ in the EVOLVE study was $4 \%$ from baseline for those treated with active compounds compared with the placebo. The rate of pulmonary exacerbation was 35\% lower in the VX-661/VX-770 group than in the placebo group (Taylor-Cousar 
et al., 2017). In the EXPAND study, the combination treatment demonstrated a mean absolute improvement of $6.8 \%$ compared with the placebo. VX-770 alone improved $\mathrm{FEV}_{1}$ only by $4.7 \%$ compared with the placebo (Rowe et al., 2017). Additionally, VX-661/VX-770 did not induce the chest tightness or drug-drug interactions observed with Orkambi ${ }^{\circledR}$. At the beginning of 2018, the FDA approved combination VX-661/VX-770 (trade name Symdeko ${ }^{\circledR}$ ) to treat CF in people ages 12 and older who have two copies of the F508del mutation and those who have at least one residual function mutation from the following: A455E, E56K, R74W, A1067T, E193K, R117C, D110E, F1052V, R347H, D110H, F1074L, R352Q, D579G, K1060T, R1070W, D1152H, L206W, S945L, D1270N, P67L, or S977F; or one following splice mutations: $711+3 \mathrm{~A} \rightarrow \mathrm{G}, 3272-26 \mathrm{~A} \rightarrow \mathrm{G}, \mathrm{E} 831 \mathrm{X}, 2789+5 \mathrm{G} \rightarrow \mathrm{A}$, or $3849+10 \mathrm{kbC} \rightarrow \mathrm{T}$. A Phase III trial is ongoing to evaluate the safety and efficacy of Symdeko ${ }^{\circledR}$ in patients ages $6-11$.

A study presented by Zawistoski et al. (2016) introduced a novel F508del-CFTR corrector-FDL169-whose potency and efficacy is comparable to VX-809. FDL169's mechanism of action could possibly be similar to that of VX-809 because combining FDL169 and VX-809 does not further increase F508del-CFTR activity. Interestingly, the inhibitory effect of VX-770 on FDL169 activity is weaker than on VX-809. This new corrector could be an alternative for VX-809.

The Galapagos Company has developed novel correctors through HTS: GLPG2222 and GLPG2851 (C1). The chemical structure of GLPG2222 (Wang et al., 2018) is similar to the structures of VX-809 and VX-661; however, it was reported to be more potent. In vitro characterization demonstrated that GLPG2222 is highly functional in primary patient cells carrying two copies of the F508del mutation. The first one-GLPG2222is currently being evaluated in a Phase II clinical study, whereas the second-GLPG2851-is currently in a Phase I study.

The modest efficacy of Orkambi ${ }^{\circledR}$ and Symdeko ${ }^{\circledR}$ triggered the development of next-generation drugs. Vertex Pharmaceuticals performed HTS on a cell model treated with VX-809 or VX-661 to search for more potent correctors, which yielded compounds that have additive rescue activity. VX-440, VX-152, VX-659, and VX-445 have been tested in separate Phase II clinical trials as a triple-combination therapy, together with VX-661 and VX-770, in adult CF patients carrying two copies of the F508del mutation or one copy of F508del and one copy of minimal CFTR function mutation. The initial results of the Phase II trials presented a significant increase in $\mathrm{FEV}_{1}$ for all groups of patients treated with the triple-combination therapy when compared with the placebo (up to $13.3 \%$ for VX-659 and $13.8 \%$ for VX-445), and a significant reduction in $\mathrm{Cl}^{-}$levels in the sweat test. The triplecombinations of VX-661/VX-770 with VX-445 or VX-659 have been tested in Phase III trials (Davies et al., 2018; Keating et al., 2018). In vitro functional tests in F508del-homozygous HBE cell cultures demonstrated that the triple combination treatment of VX-661/VX-770/VX-152 improved CFTR activity up to $\sim 75 \%$ and VX-661/VX-770//VX-440 up to $\sim 67 \%$ of normal $\mathrm{HBE}$ cells. Subsequently, HBE cells with one copy of the F508del mutation were corrected up to $\sim 47 \%$ with the triple combination containing VX-152 and up to $\sim 43 \%$ with the triple combination including VX-440. Interestingly, an important correction over
$50 \%$ of normal CFTR function was also observed in cells with genotypes "F508del/minimal function" on triple-combination regiments VX-661/VX-770VX-152, -VX-440, or -VX-659, with the highest improvement measured for VX-659.

Other next-generation correctors were introduced by Galapagos and Proteostasis Therapeutics. The initial results demonstrated that adding GLPG2737 to VX-809/VX-770 enhances the effectiveness of the treatment. The GLPG3221 (Galapagos) compound is under Phase I evaluation in healthy volunteers. Galapagos reported also that combinations of their first-generation correctors (GLPG2222 and GLPG2851) with their next-generation correctors (GLPG2737 and GLPG3221) and a potentiator GLPG1837 significantly increases $\mathrm{Cl}^{-}$transport in vitro compared with the effect of Orkambi ${ }^{\circledR}$. GLPG2222 passed early phase clinical trials and displayed improvement in potency and drug-drug interaction compared with VX809 and VX-661 (Radar, 2016). GLPG2222 and GLPG2737 correctors together with the GLPG2451 potentiator are currently being tested in Phase II clinical trials as a triple-combination therapy (FALCON).

The PTI-801 (Proteostasis Therapeutics), a third-generation corrector, showed superior in vitro efficacy over known correctors and synergy. PTI-801 in triple combination together with Orkambi ${ }^{\circledR}$ and the PTI-428 amplifier is currently in a Phase I clinical evaluation of safety and tolerability. Initial positive results have been announced by Proteostasis company (Proteostasis Announces Positive Data from Ongoing Phase 1 Study of PTI-801 in Cystic Fibrosis Patients on Background Orkambi ${ }^{\circledR}$ Therapy, 2018). Proteostasis Company reported that potentiator PTI-808 enhanced the function of mutated F508delCFTR in vitro and restored it to almost normal levels when combined with PTI-428 and PTI-801. This second triple therapy obtained fast-track status from the FDA.

Recently, Veit et al. (2018) identified new compounds-4172, 6258,3151 -stabilizing specific folding defects of F508del-CFTR. These rationally designed compounds lead to $\sim 50-100 \%$ of wild-type-level correction in immortalized and primary human airway epithelia and in mouse nasal epithelia. The strategy to use compounds that synergistically aim at distinct structural defects proved to be efficient to rescue mutant expression and function at the PM. Therefore, the combination of correctors could translate into improved clinical benefit in patients with $\mathrm{CF}$.

\section{Amplifiers}

Therapies to treat CF induced by mutations leading to decreased CFTR synthesis (Class V) requires agents that stimulate protein expression. The compounds enhancing the expression of CFTR protein, with a following increase of its quantity in the ER and the PM, are called amplifiers. Amplifiers could also be used as a therapy for other CFTR mutants when in combination with correctors and potentiators.

The PTI-428 compound from Proteostasis Therapeutics is a first-in-class CFTR amplifier that showed an in vitro increase in CFTR protein levels across genotypes. The amplifier could potentially improve mRNA stability and/or assist the processes surrounding CFTR transcription or translation. As 
reported by Molinski et al. (2017), PTI amplifier enhanced correction achieved with VX-809 and VX-770 treatment in CF cells and tissues from patients with rare CFTR mutations ( $\Delta$ I1234_R1239-CFTR).

\section{Stabilizers}

Class VI CFTR mutants and corrected Class II mutants, including F508del-CFTR localized to the PM present a reduced half-life because of increased endocytosis and decreased recycling. The instability of CFTR in the PM requires compounds that anchor mutant proteins in the membrane. Stabilizers are molecules that rectify the intrinsic protein instability and increase the CFTR residence time at the $\mathrm{PM} /$ decrease protein degradation rate from the PM.

It has been shown (Moniz et al., 2013) that hepatocytes growth factor (HGF) stimulated Rac1 signaling and contributed to F508del-CFTR anchoring at the cell surface through interactions with NHERF-1. Co-treatment of cells with HGF and lumacaftor improved the rescue of F508del-CFTR and stimulated CFTR stabilization at the apical membrane compared with lumacaftor treatment alone (Loureiro et al., 2015). An increase in CFTR interaction with NHERF-1 and subsequent stabilization of the CFTR mutant at the PM was also observed in airway cells treated with vasoactive intestinal peptide (VIP) (Rafferty et al., 2009).

Other strategies to stabilize CFTR at the PM by decreasing its endocytosis rate include cAMP signaling through EPAC1 (a guanine nucleotide exchange factor exchange protein directly activated by cAMP) (Lobo et al., 2016) and an inhibition of $S$-nitrosoglutathione reductase with $S$-nitrosylating agents, such as the endogenous $S$-nitrosoglutathione (GSNO). This latter strategy prevents CFTR interactions with Hsp70/Hsp90 chaperones (Marozkina et al., 2010; Zaman et al., 2016).

Cavosonstat (N91115, Nivalis)-an inhibitor of $S$-nitrosoglutathione reductase (GSNOR) through inhibiting GSNOR-increases $S$-nitrosoglutathione levels and leads to CFTR maturation and PM stability (Donaldson et al., 2017). A phase II clinical study is now being conducted to test Cavosonstat for patients with two copies of the F508del mutation

\section{REFERENCES}

Accurso, F. J., Rowe, S. M., Clancy, J. P., Boyle, M. P., Dunitz, J. M., Durie, P. R., et al. (2010). Effect of VX-770 in persons with cystic fibrosis and the G551DCFTR mutation. N. Engl. J. Med. 363, 1991-2003. doi: 10.1056/NEJMoa0909825

Alapati, D., and Morrisey, E. E. (2017). Gene editing and genetic lung disease. basic research meets therapeutic application. Am. J. Respir. Cell Mol. Biol. 56, 283-290. doi: 10.1165/rcmb.2016-0301PS

Aleksandrov, A. A., Aleksandrov, L., and Riordan, J. R. (2002). Nucleoside triphosphate pentose ring impact on CFTR gating and hydrolysis. FEBS Lett. 518, 183-188. doi: 10.1016/S0014-5793(02)02698-4

Alton, E. W. F. W., Armstrong, D. K., Ashby, D., Bayfield, K. J., Bilton, D., Bloomfield, E. V., et al. (2015). Repeated nebulisation of non-viral CFTR gene therapy in patients with cystic fibrosis: a randomised, double-blind, placebocontrolled, phase 2b trial. Lancet Respir. Med. 3, 684-691. doi: 10.1016/S22132600(15)00245-3 in combination therapy with VX-809/VX-770 or for patients with gating mutants and receiving VX-770.

\section{CONCLUSION}

New light has been shed on the molecular targets and pathways for therapeutic strategy thanks to the increasing comprehension of the cellular consequences of CFTR mutations.

The astonishing results of clinical trials with protein therapy demonstrate the clinical efficacy of mutationpersonalized therapy. Nowadays, scientists are convinced that an improvement of CFTR function at the molecular level can translate into an improvement in lung function and significantly improve the daily life of the patients and, most likely, their survival. It is expected that the near future will herald an era when therapeutic options will be motivated by personalized information.

Future perspectives are to develop mutation-specific and mutation-independent therapies that achieve near wild-type processing and function, as in case of the triple-combination therapy for the F508del-CFTR mutant. Further studies, however, will be needed to assess long-term efficacy and tolerance. Importantly, DNA or mRNA editing in preclinical development may allow for correct non-rescuable mutations and ultra-rare genotypes that are not targeted by current protein therapies. The next challenge is to implement those therapies in newborns with the aim of targeting the basic defect that prevents organ injury in this population.

\section{AUTHOR CONTRIBUTIONS}

IP, AG, and IS-G wrote the manuscript. $\mathrm{AH}$ and $\mathrm{AE}$ reviewed the manuscript.

\section{FUNDING}

The publication of this review has been funded by ABCF Mucoviscidose Foundation.

Awatade, N. T., Uliyakina, I., Farinha, C. M., Clarke, L. A., Mendes, K., Solé, A., et al. (2015). Measurements of functional responses in human primary lung cells as a basis for personalized therapy for cystic fibrosis. EBioMedicine 2, 147-153. doi: 10.1016/j.ebiom.2014.12.005

Bangel-Ruland, N., Tomczak, K., Fernández Fernández, E., Leier, G., Leciejewski, B., Rudolph, C., et al. (2013). Cystic fibrosis transmembrane conductance regulator-mRNA delivery: a novel alternative for cystic fibrosis gene therapy. J. Gene. Med. 15, 414-426. doi: 10.1002/jgm.2748

Bellec, J., Bacchetta, M., Losa, D., Anegon, I., Chanson, M., and Nguyen, T. H. (2015). CFTR inactivation by lentiviral vector-mediated RNA interference and CRISPR-Cas9 genome editing in human airway epithelial cells. Curr. Gene Ther. 15, 447-459. doi: 10.2174/1566523215666150812115939

Bellon, G., Michel-Calemard, L., Thouvenot, D., Jagneaux, V., Poitevin, F., Malcus, C., et al. (1997). Aerosol administration of a recombinant adenovirus expressing CFTR to cystic fibrosis patients: a phase I clinical trial. Hum. Gene Ther. 8, 15-25. doi: 10.1089/hum.1997.8.1-15 
Berger, A. L., Randak, C. O., Ostedgaard, L. S., Karp, P. H., Vermeer, D. W., and Welsh, M. J. (2005). Curcumin stimulates cystic fibrosis transmembrane conductance regulator Cl- channel activity. J. Biol. Chem. 280, 5221-5226. doi: 10.1074/jbc.M412972200

Bergougnoux, A., Petit, A., Knabe, L., Bribes, E., Chiron, R., De Sario, A., et al. (2017). The HDAC inhibitor SAHA does not rescue CFTR membrane expression in cystic fibrosis. Int. J. Biochem. Cell Biol. 88, 124-132. doi: 10.1016/ j.biocel.2017.05.002

Billet, A., Mornon, J.-P., Jollivet, M., Lehn, P., Callebaut, I., and Becq, F. (2013). CFTR: effect of ICL2 and ICL4 amino acids in close spatial proximity on the current properties of the channel. J. Cyst. Fibros. 12, 737-745. doi: 10.1016/j.jcf. 2013.02.002

Bompadre, S. G., Li, M., and Hwang, T.-C. (2008). Mechanism of G551DCFTR (cystic fibrosis transmembrane conductance regulator) potentiation by a high affinity ATP analog. J. Biol. Chem. 283, 5364-5369. doi: 10.1074/jbc. M709417200

Brewington, J. J., Filbrandt, E. T., LaRosa, F. J., Ostmann, A. J., Strecker, L. M., Szczesniak, R. D., et al. (2018). Detection of CFTR function and modulation in primary human nasal cell spheroids. J. Cyst. Fibros. 17, 26-33. doi: 10.1016/j.jcf. 2017.06.010

Budriesi, R., Ioan, P., Leoni, A., Pedemonte, N., Locatelli, A., Micucci, M., et al. (2011). Cystic fibrosis: a new target for 4-imidazo[2,1-b]thiazole-1,4dihydropyridines. J. Med. Chem. 54, 3885-3894. doi: 10.1021/jm200199r

Cai, Z., Taddei, A., and Sheppard, D. N. (2006). Differential sensitivity of the cystic fibrosis (CF)-associated mutants G551D and G1349D to potentiators of the cystic fibrosis transmembrane conductance regulator (CFTR) Cl-channel. J. Biol. Chem. 281, 1970-1977. doi: 10.1074/jbc.M510576200

Caplen, N. J., Alton, E. W., Middleton, P. G., Dorin, J. R., Stevenson, B. J., Gao, X., et al. (1995). Liposome-mediated CFTR gene transfer to the nasal epithelium of patients with cystic fibrosis. Nat. Med. 1, 39-46. doi: 10.1038/nm0195-39

Carter, S., Kelly, S., Caples, E., Grogan, B., Doyle, J., Gallagher, C. G., et al. (2015). Ivacaftor as salvage therapy in a patient with cystic fibrosis genotype F508del/R117H/IVS8-5T. J. Cyst. Fibros. 14, e4-e5. doi: 10.1016/j.jcf.2015.01. 010

Catanese, D. J., Fogg, J. M., Schrock, D. E., Gilbert, B. E., and Zechiedrich, L. (2012). Supercoiled Minivector DNA resists shear forces associated with gene therapy delivery. Gene Ther. 19, 94-100. doi: 10.1038/gt.2011.77

Cholon, D. M., Quinney, N. L., Fulcher, M. L., Esther, C. R., Das, J., Dokholyan, N. V., et al. (2014). Potentiator ivacaftor abrogates pharmacological correction of $\triangle$ F508 CFTR in cystic fibrosis. Sci. Transl. Med. 6:246ra96. doi: 10.1126/ scitranslmed. 3008680

Clancy, J. P., Rowe, S. M., Accurso, F. J., Aitken, M. L., Amin, R. S., Ashlock, M. A., et al. (2012). Results of a phase IIa study of VX-809, an investigational CFTR corrector compound, in subjects with cystic fibrosis homozygous for the F508del-CFTR mutation. Thorax 67, 12-18. doi: 10.1136/thoraxjnl-2011200393

Colas, J., Faure, G., Saussereau, E., Trudel, S., Rabeh, W. M., Bitam, S., et al. (2012). Disruption of cytokeratin-8 interaction with F508del-CFTR corrects its functional defect. Hum. Mol. Genet. 21, 623-634. doi: 10.1093/hmg/ ddr496

Conese, M., Beccia, E., Castellani, S., Di Gioia, S., Colombo, C., Angiolillo, A., et al. (2018). The long and winding road: stem cells for cystic fibrosis. Expert Opin. Biol. Ther. 18, 281-292. doi: 10.1080/14712598.2018.1413087

Crane, A. M., Kramer, P., Bui, J. H., Chung, W. J., Li, X. S., Gonzalez-Garay, M. L., et al. (2015). Targeted correction and restored function of the CFTR gene in cystic fibrosis induced pluripotent stem cells. Stem Cell Rep. 4, 569-577. doi: 10.1016/j.stemcr.2015.02.005

Crosby, J. R., Zhao, C., Jiang, C., Bai, D., Katz, M., Greenlee, S., et al. (2017). Inhaled $\mathrm{ENaC}$ antisense oligonucleotide ameliorates cystic fibrosis-like lung disease in mice. J. Cyst. Fibros. 16, 671-680. doi: 10.1016/j.jcf.2017.05.003

Crystal, R. G., McElvaney, N. G., Rosenfeld, M. A., Chu, C. S., Mastrangeli, A., Hay, J. G., et al. (1994). Administration of an adenovirus containing the human CFTR cDNA to the respiratory tract of individuals with cystic fibrosis. Nat. Genet. 8, 42-51. doi: 10.1038/ng0994-42

Davies, J. C., Moskowitz, S. M., Brown, C., Horsley, A., Mall, M. A., McKone, E. F., et al. (2018). VX-659-tezacaftor-ivacaftor in patients with cystic fibrosis and one or two Phe508del alleles. N. Engl. J. Med. 379, 1599-1611. doi: 10.1056/ NEJMoa1807119
Davis, P. B., Drumm, M., and Konstan, M. W. (1996). Cystic fibrosis. Am. J. Respir. Crit. Care Med. 154, 1229-1256. doi: 10.1164/ajrccm.154.5.8912731

De Boeck, K., Zolin, A., Cuppens, H., Olesen, H. V., and Viviani, L. (2014). The relative frequency of CFTR mutation classes in European patients with cystic fibrosis. J. Cyst. Fibros. 13, 403-409. doi: 10.1016/j.jcf.2013.12.003

Dekkers, J. F., Berkers, G., Kruisselbrink, E., Vonk, A., de Jonge, H. R., Janssens, H. M., et al. (2016). Characterizing responses to CFTR-modulating drugs using rectal organoids derived from subjects with cystic fibrosis. Sci. Transl. Med. 8:344ra84. doi: 10.1126/scitranslmed.aad8278

Dekkers, J. F., Wiegerinck, C. L., de Jonge, H. R., Bronsveld, I., Janssens, H. M., de Winter-de Groot, K. M., et al. (2013). A functional CFTR assay using primary cystic fibrosis intestinal organoids. Nat. Med. 19, 939-945. doi: 10.1038/nm. 3201

Dhand, R. (2017). Inhaled drug therapy 2016: the year in review. Respir. Care 62, 978-996. doi: 10.4187/respcare.05624

Dismuke, D., Tenenbaum, L., and Samulski, R. (2014). Biosafety of recombinant adeno-associated virus vectors. Curr. Gene Ther. 13, 434-452. doi: 10.2174/ 15665232113136660007

Donaldson, S. H., Solomon, G. M., Zeitlin, P. L., Flume, P. A., Casey, A., McCoy, K., et al. (2017). Pharmacokinetics and safety of cavosonstat (N91115) in healthy and cystic fibrosis adults homozygous for F508DEL-CFTR. J. Cyst. Fibros. 16, 371-379. doi: 10.1016/j.jcf.2017.01.009

Drumm, M. L., Pope, H. A., Cliff, W. H., Rommens, J. M., Marvin, S. A., Tsui, L. C., et al. (1990). Correction of the cystic fibrosis defect in vitro by retrovirusmediated gene transfer. Cell 62, 1227-1233. doi: 10.1016/0092-8674(90) 90398-X

Drumm, M. L., Wilkinson, D. J., Smit, L. S., Worrell, R. T., Strong, T. V., Frizzell, R. A., et al. (1991). Chloride conductance expressed by delta F508 and other mutant CFTRs in Xenopus oocytes. Science 254, 1797-1799. doi: 10.1126/ science. 1722350

Du, K., Sharma, M., and Lukacs, G. L. (2005). The DeltaF508 cystic fibrosis mutation impairs domain-domain interactions and arrests post-translational folding of CFTR. Nat. Struct. Mol. Biol. 12, 17-25. doi: 10.1038/nsmb882

Eckford, P. D. W., Li, C., Ramjeesingh, M., and Bear, C. E. (2012). Cystic fibrosis transmembrane conductance regulator (CFTR) potentiator VX-770 (Ivacaftor) opens the defective channel gate of mutant CFTR in a phosphorylationdependent but ATP-independent manner. J. Biol. Chem. 287, 36639-36649. doi: 10.1074/jbc.M112.393637

Eckford, P. D. W., Ramjeesingh, M., Molinski, S., Pasyk, S., Dekkers, J. F., Li, C., et al. (2014). VX-809 and related corrector compounds exhibit secondary activity stabilizing active F508del-CFTR after its partial rescue to the cell surface. Chem. Biol. 21, 666-678. doi: 10.1016/j.chembiol.2014.02.021

Egan, M. E., Pearson, M., Weiner, S. A., Rajendran, V., Rubin, D., GlöcknerPagel, J., et al. (2004). Curcumin, a major constituent of turmeric, corrects cystic fibrosis defects. Science 304, 600-602. doi: 10.1126/science.10 93941

Farinha, C. M., King-Underwood, J., Sousa, M., Correia, A. R., Henriques, B. J., Roxo-Rosa, M., et al. (2013). Revertants, low temperature, and correctors reveal the mechanism of F508del-CFTR rescue by VX-809 and suggest multiple agents for full correction. Chem. Biol. 20, 943-955. doi: 10.1016/j.chembiol.2013.06. 004

Farinha, C. M., Sousa, M., Canato, S., Schmidt, A., Uliyakina, I., and Amaral, M. D. (2015). Increased efficacy of VX-809 in different cellular systems results from an early stabilization effect of F508del-CFTR. Pharmacol. Res. Perspect. 3:e00152. doi: $10.1002 /$ prp2.152

Faure, G., Bakouh, N., Lourdel, S., Odolczyk, N., Premchandar, A., Servel, N., et al. (2016). Rattlesnake phospholipase A2 increases CFTR-chloride channel current and corrects $\triangle$ F508CFTR dysfunction: impact in cystic fibrosis. J. Mol. Biol. 428, 2898-2915. doi: 10.1016/j.jmb.2016.05.016

Fidler, M. C., Beusmans, J., Panorchan, P., and Van Goor, F. (2017). Correlation of sweat chloride and percent predicted $\mathrm{FEV}_{1}$ in cystic fibrosis patients treated with ivacaftor. J. Cyst. Fibros. 16, 41-44. doi: 10.1016/j.jcf.2016.10.002

Firth, A. L., Menon, T., Parker, G. S., Qualls, S. J., Lewis, B. M., Ke, E., et al. (2015) Functional gene correction for cystic fibrosis in lung epithelial cells generated from patient iPSCs. Cell Rep. 12, 1385-1390. doi: 10.1016/j.celrep.2015.07.062

Flume, P. A., Liou, T. G., Borowitz, D. S., Li, H., Yen, K., Ordoñez, C. L., et al. (2012). Ivacaftor in subjects with cystic fibrosis who are homozygous for the F508del-CFTR mutation. Chest 142, 718-724. doi: 10.1378/chest.11-2672 
Foldvari, M., Chen, D. W., Nafissi, N., Calderon, D., Narsineni, L., and Rafiee, A. (2016). Non-viral gene therapy: gains and challenges of non-invasive administration methods. J. Controll. Release 240, 165-190. doi: 10.1016/j. jconrel.2015.12.012

French, P. J., Bijman, J., Bot, A. G., Boomaars, W. E., Scholte, B. J., and de Jonge, H. R. (1997). Genistein activates CFTR Cl- channels via a tyrosine kinase- and protein phosphatase-independent mechanism. Am. J. Physiol. Cell Physiol. 273, C747-C753. doi: 10.1152/ajpcell.1997.273.2.C747

Friedman, K. J., Kole, J., Cohn, J. A., Knowles, M. R., Silverman, L. M., and Kole, R. (1999). Correction of aberrant splicing of the cystic fibrosis transmembrane conductance regulator (CFTR) gene by antisense oligonucleotides. J. Biol. Chem. 274, 36193-36199. doi: 10.1074/jbc.274.51.36193

Gelman, M. S., Kannegaard, E. S., and Kopito, R. R. (2002). A principal role for the proteasome in endoplasmic reticulum-associated degradation of misfolded intracellular cystic fibrosis transmembrane conductance regulator. J. Biol. Chem. 277, 11709-11714. doi: 10.1074/jbc.M111958200

Gentzsch, M., Ren, H. Y., Houck, S. A., Quinney, N. L., Cholon, D. M., Sopha, P., et al. (2016). Restoration of R117H CFTR folding and function in human airway cells through combination treatment with VX-809 and VX-770. Am. J. Physiol. Lung Cell. Mol. Physiol. 311, L550-L559. doi: 10.1152/ajplung.001 86.2016

Goor, F. V., Hadida, S., Grootenhuis, P. D. J., Burton, B., Cao, D., Neuberger, T., et al. (2009). Rescue of CF airway epithelial cell function in vitro by a CFTR potentiator, VX-770. Proc. Natl. Acad. Sci. U.S.A. 106, 18825-18830. doi: 10. 1073/pnas.0904709106

Gregory, L. G., Harbottle, R. P., Lawrence, L., Knapton, H. J., Themis, M., and Coutelle, C. (2003). Enhancement of adenovirus-mediated gene transfer to the airways by DEAE dextran and sodium caprate in vivo. Mol. Ther. 7, 19-26. doi: 10.1016/S1525-0016(02)00021-7

Guimbellot, J. S., Leach, J. M., Chaudhry, I. G., Quinney, N. L., Boyles, S. E., Chua, M., et al. (2018). Nasospheroids permit measurements of CFTRdependent fluid transport. JCI Insight doi: 10.1172/jci.insight.95734 [Epub ahead of print].

Guo, L., Karoubi, G., Duchesneau, P., Shutova, M. V., Sung, H.-K., Tonge, P., et al. (2017). Generation of induced progenitor-like cells from mature epithelial cells using interrupted reprogramming. Stem Cell Rep. 9, 1780-1795. doi: 10.1016/j. stemcr.2017.10.022

Hagemeijer, M. C., Siegwart, D. J., Strug, L. J., Cebotaru, L., Torres, M. J., Sofoluwe, A., et al. (2018). Translational research to enable personalized treatment of cystic fibrosis. J. Cyst. Fibros. 17, S46-S51. doi: 10.1016/j.jcf.2017. 10.017

Hart, S. L., and Harrison, P. T. (2017). Genetic therapies for cystic fibrosis lung disease. Curr. Opin. Pharmacol. 34, 119-124. doi: 10.1016/j.coph.2017.10.006

Harvey, B.-G., Maroni, J., O’Donoghue, K. A., Chu, K. W., Muscat, J. C., Pippo, A. L., et al. (2002). Safety of local delivery of low- and intermediatedose adenovirus gene transfer vectors to individuals with a spectrum of morbid conditions. Hum. Gene Ther. 13, 15-63. doi: 10.1089/1043034015271 2638

Hay, J. G., McElvaney, N. G., Herena, J., and Crystal, R. G. (1995). Modification of nasal epithelial potential differences of individuals with cystic fibrosis consequent to local administration of a normal CFTR cDNA adenovirus gene transfer vector. Hum. Gene Ther. 6, 1487-1496. doi: 10.1089/hum.1995.6.111487

He, L., Kota, P., Aleksandrov, A. A., Cui, L., Jensen, T., Dokholyan, N. V., et al. (2012). Correctors of $\triangle$ F508 CFTR restore global conformational maturation without thermally stabilizing the mutant protein. FASEB J. 27, 536-545. doi: 10.1096/fj.12-216119

Hollywood, J. A., Lee, C. M., Scallan, M. F., and Harrison, P. T. (2016). Analysis of gene repair tracts from Cas9/gRNA double-stranded breaks in the human CFTR gene. Sci. Rep. 6:32230. doi: 10.1038/srep32230

Howard, M., Frizzell, R. A., and Bedwell, D. M. (1996). Aminoglycoside antibiotics restore CFTR function by overcoming premature stop mutations. Nat. Med. 2, 467-469. doi: 10.1038/nm0496-467

Hutt, D. M., Herman, D., Rodrigues, A. P. C., Noel, S., Pilewski, J. M., Matteson, J., et al. (2010). Reduced histone deacetylase 7 activity restores function to misfolded CFTR in cystic fibrosis. Nat. Chem. Biol. 6, 25-33. doi: 10.1038/ nchembio. 275
Hwang, T.-C., and Sheppard, D. N. (2009). Gating of the CFTR Cl- channel by ATP-driven nucleotide-binding domain dimerisation. J. Physiol. 587, 21512161. doi: 10.1113/jphysiol.2009.171595

Hwang, T. C., Wang, F., Yang, I. C., and Reenstra, W. W. (1997). Genistein potentiates wild-type and delta F508-CFTR channel activity. Am. J. Physiol. Cell Physiol. 273, C988-C998. doi: 10.1152/ajpcell.1997.273. 3.C988

Igreja, S., Clarke, L. A., Botelho, H. M., Marques, L., and Amaral, M. D. (2016). Correction of a cystic fibrosis splicing mutation by antisense oligonucleotides. Hum. Mutat. 37, 209-215. doi: 10.1002/humu.22931

Illek, B., and Fischer, H. (1998). Flavonoids stimulate $\mathrm{Cl}$ conductance of human airway epithelium in vitro and in vivo. Am. J. Physiol. Lung Cell. Mol. Physiol. 275, L902-L910. doi: 10.1152/ajplung.1998.275.5.L902

Illek, B., Fischer, H., and Machen, T. E. (1996). Alternate stimulation of apical CFTR by genistein in epithelia. Am. J. Physiol. Cell Physiol. 270, C265-C275. doi: 10.1152/ajpcell.1996.270.1.C265

Illek, B., Fischer, H., Santos, G. F., Widdicombe, J. H., Machen, T. E., and Reenstra, W. W. (1995). cAMP-independent activation of CFTR Cl channels by the tyrosine kinase inhibitor genistein. Am. J. Physiol. Cell Physiol. 268, C886-C893. doi: 10.1152/ajpcell.1995.268.4.C886

Jensen, T. J., Loo, M. A., Pind, S., Williams, D. B., Goldberg, A. L., and Riordan, J. R. (1995). Multiple proteolytic systems, including the proteasome, contribute to CFTR processing. Cell 83, 129-135. doi: 10.1016/0092-8674(95)90241-4

Jih, K.-Y., and Hwang, T.-C. (2013). Vx-770 potentiates CFTR function by promoting decoupling between the gating cycle and ATP hydrolysis cycle. Proc. Natl. Acad. Sci. U.S.A. 110, 4404-4409. doi: 10.1073/pnas.1215982110

Joseph, P. M., O’Sullivan, B. P., Lapey, A., Dorkin, H., Oren, J., Balfour, R., et al. (2001). Aerosol and lobar administration of a recombinant adenovirus to individuals with cystic fibrosis. I. Methods, safety, and clinical implications. Hum. Gene Ther. 12, 1369-1382. doi: 10.1089/104303401750298535

Kalid, O., Mense, M., Fischman, S., Shitrit, A., Bihler, H., Ben-Zeev, E., et al. (2010). Small molecule correctors of F508del-CFTR discovered by structurebased virtual screening. J. Comput. Aided Mol. Des. 24, 971-991. doi: 10.1007/ s10822-010-9390-0

Kandasamy, J., Atia-Glikin, D., Shulman, E., Shapira, K., Shavit, M., Belakhov, V., et al. (2012). Increased selectivity towards cytoplasmic versus mitochondrial ribosome confers improved efficiency of synthetic aminoglycosides in fixing damaged genes: a strategy for treatment of genetic diseases caused by nonsense mutations. J. Med. Chem. 55, 10630-10643. doi: 10.1021/jm3012992

Katkin, J. P., Gilbert, B. E., Langston, C., French, K., and Beaudet, A. L. (1995). Aerosol delivery of a beta-galactosidase adenoviral vector to the lungs of rodents. Hum. Gene Ther. 6, 985-995. doi: 10.1089/hum.1995.6.8-985

Keating, D., Marigowda, G., Burr, L., Daines, C., Mall, M. A., McKone, E. F., et al. (2018). VX-445-tezacaftor-ivacaftor in patients with cystic fibrosis and one or two Phe508del alleles. N. Engl. J. Med. 379, 1612-1620. doi: 10.1056/ NEJMoa1807120

Kerem, E., Hirawat, S., Armoni, S., Yaakov, Y., Shoseyov, D., Cohen, M., et al. (2008). Effectiveness of PTC124 treatment of cystic fibrosis caused by nonsense mutations: a prospective phase II trial. Lancet 372, 719-727. doi: 10.1016/ S0140-6736(08)61168-X

Kerem, E., Konstan, M. W., De Boeck, K., Accurso, F. J., Sermet-Gaudelus, I., Wilschanski, M., et al. (2014). Ataluren for the treatment of nonsense-mutation cystic fibrosis: a randomised, double-blind, placebo-controlled phase 3 trial. Lancet Respir. Med. 2, 539-547. doi: 10.1016/S2213-2600(14)70100-6

Knowles, M. R., Hohneker, K. W., Zhou, Z., Olsen, J. C., Noah, T. L., Hu, P. C., et al. (1995). A controlled study of adenoviral-vector-mediated gene transfer in the nasal epithelium of patients with cystic fibrosis. N. Engl. J. Med. 333, 823-831. doi: 10.1056/NEJM199509283331302

Konstan, M. W., McKone, E. F., Moss, R. B., Marigowda, G., Tian, S., Waltz, D., et al. (2017). Assessment of safety and efficacy of long-term treatment with combination lumacaftor and ivacaftor therapy in patients with cystic fibrosis homozygous for the F508del-CFTR mutation (PROGRESS): a phase 3, extension study. Lancet Respir. Med. 5, 107-118. doi: 10.1016/S2213-2600(16) 30427-1

Kuhn, A. N., Beißert, T., Simon, P., Vallazza, B., Buck, J., Davies, B. P., et al. (2012). mRNA as a versatile tool for exogenous protein expression. Curr. Gene Ther. 12, 347-361. doi: 10.2174/156652312802762536 
LaRusch, J., Jung, J., General, I. J., Lewis, M. D., Park, H. W., Brand, R. E., et al. (2014). Mechanisms of CFTR functional variants that impair regulated bicarbonate permeation and increase risk for pancreatitis but not for cystic fibrosis. PLoS Genet. 10:e1004376. doi: 10.1371/journal.pgen.1004376

Lentz, Y. K., Worden, L. R., Anchordoquy, T. J., and Lengsfeld, C. S. (2005). Effect of jet nebulization on DNA: identifying the dominant degradation mechanism and mitigation methods. J. Aerosol Sci. 36, 973-990. doi: 10.1016/j.jaerosci.2004. 11.017

Linsdell, P. (2014). Functional architecture of the CFTR chloride channel. Mol. Membr. Biol. 31, 1-16. doi: 10.3109/09687688.2013.868055

Li, L., He, Z.-Y., Wei, X.-W., Gao, G.-P., and Wei, Y.-Q. (2015). Challenges in CRISPR/CAS9 delivery: potential roles of nonviral vectors. Hum. Gene Ther. 26, 452-462. doi10.1089/hum.2015.069

Lipecka, J., Norez, C., Bensalem, N., Baudouin-Legros, M., Planelles, G., Becq, F., et al. (2006). Rescue of DeltaF508-CFTR (cystic fibrosis transmembrane conductance regulator) by curcumin: involvement of the keratin 18 network. J. Pharmacol. Exp. Ther. 317, 500-505. doi: 10.1124/jpet.105.097667

Lobo, M. J., Amaral, M. D., Zaccolo, M., and Farinha, C. M. (2016). EPAC1 activation by cAMP stabilizes CFTR at the membrane by promoting its interaction with NHERF1. J. Cell Sci. 129, 2599-2612. doi: 10.1242/jcs.185629

Loo, T. W., Bartlett, M. C., and Clarke, D. M. (2008). Correctors promote folding of the CFTR in the endoplasmic reticulum. Biochem. J. 413, 29-36. doi: 10.1042/ BJ20071690

Loo, T. W., Bartlett, M. C., and Clarke, D. M. (2009). Correctors enhance maturation of $\triangle F 508$ CFTR by promoting interactions between the two halves of the molecule. Biochemistry 48, 9882-9890. doi: 10.1021/bi9004842

Loo, T. W., Bartlett, M. C., and Clarke, D. M. (2013). Corrector VX-809 stabilizes the first transmembrane domain of CFTR. Biochem. Pharmacol. 86, 612-619. doi: 10.1016/j.bcp.2013.06.028

Loo, T. W., and Clarke, D. M. (2017). Corrector VX-809 promotes interactions between cytoplasmic loop one and the first nucleotide-binding domain of CFTR. Biochem. Pharmacol. 136, 24-31. doi: 10.1016/j.bcp.2017.03.020

Loureiro, C. A., Matos, A. M., Dias-Alves, Â, Pereira, J. F., Uliyakina, I., Barros, P., et al. (2015). A molecular switch in the scaffold NHERF1 enables misfolded CFTR to evade the peripheral quality control checkpoint. Sci. Signal. 8:ra48. doi: 10.1126/scisignal.aaa1580

Lubamba, B., Lecourt, H., Lebacq, J., Lebecque, P., De Jonge, H., Wallemacq, P., et al. (2008). Preclinical evidence that sildenafil and vardenafil activate chloride transport in cystic fibrosis. Am. J. Respir. Crit. Care Med. 177, 506-515. doi: 10.1164/rccm.200703-344OC

Lukacs, G. L., Chang, X. B., Bear, C., Kartner, N., Mohamed, A., Riordan, J. R., et al. (1993). The delta F508 mutation decreases the stability of cystic fibrosis transmembrane conductance regulator in the plasma membrane. Determination of functional half-lives on transfected cells. J. Biol. Chem. 268, 21592-21598.

Lukacs, G. L., and Verkman, A. S. (2012). CFTR: folding, misfolding and correcting the $\Delta$ F508 conformational defect. Trends Mol. Med. 18, 81-91. doi: 10.1016/j. molmed.2011.10.003

Maquat, L. E. (2004). Nonsense-mediated mRNA decay: splicing, translation and mRNP dynamics. Nat. Rev. Mol. Cell Biol. 5, 89-99. doi: 10.1038/nrm1310

Marozkina, N. V., Yemen, S., Borowitz, M., Liu, L., Plapp, M., Sun, F., et al. (2010). Hsp 70/Hsp 90 organizing protein as a nitrosylation target in cystic fibrosis therapy. Proc. Natl. Acad. Sci. U.S.A. 107, 11393-11398. doi: 10.1073/pnas. 0909128107

Martin, U. (2017). Therapeutic application of pluripotent stem cells: challenges and risks. Front. Med. 4:229. doi: 10.3389/fmed.2017.00229

Martiniano, S. L., Sagel, S. D., and Zemanick, E. T. (2016). Cystic fibrosis: a model system for precision medicine. Curr. Opin. Pediatr. 28, 312-317. doi: 10.1097/ MOP.0000000000000351

Mastorakos, P., da Silva, A. L., Chisholm, J., Song, E., Choi, W. K., Boyle, M. P., et al. (2015). Highly compacted biodegradable DNA nanoparticles capable of overcoming the mucus barrier for inhaled lung gene therapy. Proc. Natl. Acad. Sci. U.S.A. 112, 8720-8725. doi: 10.1073/pnas. 1502281112

McQualter, J. L., Yuen, K., Williams, B., and Bertoncello, I. (2010). Evidence of an epithelial stem/progenitor cell hierarchy in the adult mouse lung. Proc. Natl. Acad. Sci. U.S.A. 107, 1414-1419. doi: 10.1073/pnas.0909207107

Miki, H., Zhou, Z., Li, M., Hwang, T.-C., and Bompadre, S. G. (2010). Potentiation of disease-associated cystic fibrosis transmembrane conductance regulator mutants by hydrolyzable ATP analogs. J. Biol. Chem. 285, 19967-19975. doi: 10.1074/jbc.M109.092684

Molinski, S. V., Ahmadi, S., Ip, W., Ouyang, H., Villella, A., Miller, J. P., et al. (2017). Orkambi ${ }^{\circledR}$ and amplifier co-therapy improves function from a rare CFTR mutation in gene-edited cells and patient tissue. EMBO Mol. Med. 9, 1224-1243. doi: 10.15252/emmm.201607137

Moniz, S., Sousa, M., Moraes, B. J., Mendes, A. I., Palma, M., Barreto, C., et al. (2013). HGF stimulation of Racl signaling enhances pharmacological correction of the most prevalent cystic fibrosis mutant F508del-CFTR. ACS Chem. Biol. 8, 432-442. doi: 10.1021/cb300484r

Mottais, A., Le Gall, T., Sibiril, Y., Ravel, J., Laurent, V., d'Arbonneau, F., et al. (2017). Enhancement of lung gene delivery after aerosol: a new strategy using non-viral complexes with antibacterial properties. Biosci. Rep. 37:BSR20160618. doi: 10.1042/BSR20160618

Muallem, D., and Vergani, P. (2009). Review. ATP hydrolysis-driven gating in cystic fibrosis transmembrane conductance regulator. Philos. Trans. R. Soc. Lond. B Biol. Sci. 364, 247-255. doi: 10.1098/rstb.2008.0191

Nichols, D. P., and Chmiel, J. F. (2015). Inflammation and its genesis in cystic fibrosis. Pediatr. Pulmonol. 50, S39-S56. doi: 10.1002/ppul.23242

Noël, S., Wilke, M., Bot, A. G. M., De Jonge, H. R., and Becq, F. (2008). Parallel improvement of sodium and chloride transport defects by miglustat (n-butyldeoxynojyrimicin) in cystic fibrosis epithelial cells. J. Pharmacol. Exp. Ther. 325, 1016-1023. doi: 10.1124/jpet.107.135582

Norez, C., Antigny, F., Noel, S., Vandebrouck, C., and Becq, F. (2009). A cystic fibrosis respiratory epithelial cell chronically treated by miglustat acquires a non-cystic fibrosis-like phenotype. Am. J. Respir. Cell Mol. Biol. 41, 217-225. doi: $10.1165 / \mathrm{rcmb} .2008-0285 \mathrm{OC}$

Nudelman, I., Rebibo-Sabbah, A., Cherniavsky, M., Belakhov, V., Hainrichson, M., Chen, F., et al. (2009). Development of novel aminoglycoside (NB54) with reduced toxicity and enhanced suppression of disease-causing premature stop mutations. J. Med. Chem. 52, 2836-2845. doi: 10.1021/jm801640k

Odolczyk, N., Fritsch, J., Norez, C., Servel, N., da Cunha, M. F., Bitam, S., et al. (2013). Discovery of novel potent $\triangle$ F508-CFTR correctors that target the nucleotide binding domain. EMBO Mol. Med. 5, 1484-1501. doi: 10.1002/ emmm.201302699

Okiyoneda, T., Veit, G., Dekkers, J. F., Bagdany, M., Soya, N., Xu, H., et al. (2013). Mechanism-based corrector combination restores $\triangle$ F508-CFTR folding and function. Nat. Chem. Biol. 9, 444-454. doi: 10.1038/nchembio.1253

Osman, G., Rodriguez, J., Chan, S. Y., Chisholm, J., Duncan, G., Kim, N., et al. (2018). PEGylated enhanced cell penetrating peptide nanoparticles for lung gene therapy. J. Control. Release 285, 35-45. doi: 10.1016/j.jconrel.2018.07.001

O’Sullivan, B. P., and Freedman, S. D. (2009). Cystic fibrosis. Lancet 373, 18911904. doi: 10.1016/S0140-6736(09)60327-5

Pasyk, S., Li, C., Ramjeesingh, M., and Bear, C. E. (2009). Direct interaction of a small-molecule modulator with G551D-CFTR, a cystic fibrosis-causing mutation associated with severe disease. Biochem. J. 418, 185-190. doi: 10.1042/ BJ20081424

Pedemonte, N., Lukacs, G. L., Du, K., Caci, E., Zegarra-Moran, O., Galietta, L. J. V., et al. (2005a). Small-molecule correctors of defective $\Delta$ F508-CFTR cellular processing identified by high-throughput screening. J. Clin. Invest. 115, 2564-2571. doi: 10.1172/JCI24898

Pedemonte, N., Sonawane, N. D., Taddei, A., Hu, J., Zegarra-Moran, O., Suen, Y. F., et al. (2005b). Phenylglycine and sulfonamide correctors of defective delta F508 and G551D cystic fibrosis transmembrane conductance regulator chloride-channel gating. Mol. Pharmacol. 67, 1797-1807. doi: 10.1124/mol.105. 010959

Pollard, B. S., and Pollard, H. B. (2018). Induced pluripotent stem cells for treating cystic fibrosis: state of the science. Pediatr. Pulmonol. 53, S12-S29. doi10.1002/ppul.24118

Popp, M. W., and Maquat, L. E. (2016). Leveraging rules of nonsense-mediated mRNA decay for genome engineering and personalized medicine. Cell 165, 1319-1322. doi: 10.1016/j.cell.2016.05.053

Pranke, I., Bidou, L., Martin, N., Blanchet, S., Hatton, A., Karri, S., et al. (2018). Factors influencing readthrough therapy for frequent cystic fibrosis premature termination codons. ERJ Open Res. 4:00080-2017. doi: 10.1183/23120541. 00080-2017

Pranke, I. M., Hatton, A., Simonin, J., Jais, J. P., Le Pimpec-Barthes, F., Carsin, A., et al. (2017). Correction of CFTR function in nasal epithelial cells from 
cystic fibrosis patients predicts improvement of respiratory function by CFTR modulators. Sci. Rep. 7:7375. doi: 10.1038/s41598-017-07504-1

Pranke, I. M., and Sermet-Gaudelus, I. (2014). Biosynthesis of cystic fibrosis transmembrane conductance regulator. Int. J. Biochem. Cell Biol. 52, 26-38. doi: 10.1016/j.biocel.2014.03.020

Pringle, I. A., Hyde, S. C., and Gill, D. R. (2009). Non-viral vectors in cystic fibrosis gene therapy: recent developments and future prospects. Expert Opin. Biol. Ther. 9, 991-1003. doi: 10.1517/14712590903055029

Proteostasis Announces Positive Data from Ongoing Phase 1 Study of PTI801 in Cystic Fibrosis Patients on Background Orkambi ${ }^{\circledR}$ Therapy (2018). Proteostasis Announces Positive Data from Ongoing Phase 1 Study of PTI801 in Cystic Fibrosis Patients on Background Orkambi ${ }^{\circledR}$ Therapy Proteostasis Therapeutics. Available at: /news-releases/news-release-details/proteostasisannounces-positive-data-ongoing-phase-1-study-pti [accessed September 21, 2018].

Pyle, L. C., Ehrhardt, A., Mitchell, L. H., Fan, L., Ren, A., Naren, A. P., et al. (2011). Regulatory domain phosphorylation to distinguish the mechanistic basis underlying acute CFTR modulators. Am. J. Physiol. Lung Cell. Mol. Physiol. 301, L587-L597. doi: 10.1152/ajplung.00465.2010

Radar, B. (2016). Galapagos \$GLPG Abstract from \#NACFC2016: Discovery of Corrector ABBV/GLPG 2222pic.twitter.com/i9RbbZBOkq. @biotechradar. Available at: https://twitter.com/biotechradar/status/778661229686562817 [accessed September 20, 2018].

Rafferty, S., Alcolado, N., Norez, C., Chappe, F., Pelzer, S., Becq, F., et al. (2009). Rescue of functional F508del cystic fibrosis transmembrane conductance regulator by vasoactive intestinal peptide in the human nasal epithelial cell line JME/CF15. J. Pharmacol. Exp. Ther. 331, 2-13. doi: 10.1124/jpet.109. 155341

Ramalho, A. S., Lewandowska, M. A., Farinha, C. M., Mendes, F., Gonçalves, J., Barreto, C., et al. (2009). Deletion of CFTR translation start site reveals functional isoforms of the protein in CF patients. Cell. Physiol. Biochem. 24, 335-346. doi: 10.1159/000257426

Ramsey, B. W., Davies, J., McElvaney, N. G., Tullis, E., Bell, S. C., Døevínek, P., et al. (2011). A CFTR potentiator in patients with cystic fibrosis and the G551D mutation. N. Engl. J. Med. 365, 1663-1672. doi: 10.1056/NEJMoa1105185

Ratjen, F., Hug, C., Marigowda, G., Tian, S., Huang, X., Stanojevic, S., et al. (2017). Efficacy and safety of lumacaftor and ivacaftor in patients aged 6-11 years with cystic fibrosis homozygous for F508del-CFTR: a randomised, placebocontrolled phase 3 trial. Lancet Respir. Med. 5, 557-567. doi: 10.1016/S22132600(17)30215-1

Ren, H. Y., Grove, D. E., De La Rosa, O., Houck, S. A., Sopha, P., Van Goor, F., et al. (2013). VX-809 corrects folding defects in cystic fibrosis transmembrane conductance regulator protein through action on membranespanning domain 1. Mol. Biol. Cell 24, 3016-3024. doi: 10.1091/mbc.e1305- 0240

Riordan, J. R. (2008). CFTR function and prospects for therapy. Annu. Rev. Biochem. 77, 701-726. doi: 10.1146/annurev.biochem.75.103004.142532

Robert, R., Carlile, G. W., Liao, J., Balghi, H., Lesimple, P., Liu, N., et al. (2010). Correction of the Delta phe508 cystic fibrosis transmembrane conductance regulator trafficking defect by the bioavailable compound glafenine. Mol. Pharmacol. 77, 922-930. doi: 10.1124/mol.109.062679

Robert, R., Carlile, G. W., Pavel, C., Liu, N., Anjos, S. M., Liao, J., et al. (2008). Structural analog of sildenafil identified as a novel corrector of the F508delCFTR trafficking defect. Mol. Pharmacol. 73, 478-489. doi: 10.1124/mol.107. 040725

Robinson, E., MacDonald, K. D., Slaughter, K., McKinney, M., Patel, S., Sun, C., et al. (2018). Lipid nanoparticle-delivered chemically modified mRNA restores chloride secretion in cystic fibrosis. Mol. Ther. 26, 2034-2046. doi: 10.1016/j. ymthe.2018.05.014

Rock, J., and Königshoff, M. (2012). Endogenous lung regeneration. Am. J. Respir. Crit. Care Med. 186, 1213-1219. doi: 10.1164/rccm.201207-1151PP

Rosenfeld, M. A., Yoshimura, K., Trapnell, B. C., Yoneyama, K., Rosenthal, E. R., Dalemans, W., et al. (1992). In vivo transfer of the human cystic fibrosis transmembrane conductance regulator gene to the airway epithelium. Cell 68, 143-155. doi: 10.1016/0092-8674(92)90213-V

Rowe, S. M., Daines, C., Ringshausen, F. C., Kerem, E., Wilson, J., Tullis, E., et al. (2017). Tezacaftor-ivacaftor in residual-function heterozygotes with cystic fibrosis. N. Engl. J. Med. 377, 2024-2035. doi: 10.1056/NEJMoa1709847
Rowe, S. M., Miller, S., and Sorscher, E. J. (2005). Cystic fibrosis. N. Engl. J. Med. 352, 1992-2001. doi: 10.1056/NEJMra043184

Rowe, S. M., Sloane, P., Tang, L. P., Backer, K., Mazur, M., BuckleyLanier, J., et al. (2011). Suppression of CFTR premature termination codons and rescue of CFTR protein and function by the synthetic aminoglycoside NB54. J. Mol. Med. 89, 1149-1161. doi: 10.1007/s00109-0110787-6

Rubenstein, R. C., and Zeitlin, P. L. (2000). Sodium 4-phenylbutyrate downregulates Hsc70: implications for intracellular trafficking of DeltaF508CFTR. Am. J. Physiol. Cell Physiol. 278, C259-C267. doi: 10.1152/ajpcell.2000. 278.2.C259

Sampson, H. M., Robert, R., Liao, J., Matthes, E., Carlile, G. W., Hanrahan, J. W., et al. (2011). Identification of a NBD1-binding pharmacological chaperone that corrects the trafficking defect of F508del-CFTR. Chem. Biol. 18, 231-242. doi: 10.1016/j.chembiol.2010.11.016

Sanz, D. J., Hollywood, J. A., Scallan, M. F., and Harrison, P. T. (2017). Cas9/gRNA targeted excision of cystic fibrosis-causing deep-intronic splicing mutations restores normal splicing of CFTR mRNA. PLoS One 12:e0184009. doi: 10.1371/ journal.pone.0184009

Scaria, A., St George, J. A., Jiang, C., Kaplan, J. M., Wadsworth, S. C., and Gregory, R. J. (1998). Adenovirus-mediated persistent cystic fibrosis transmembrane conductance regulator expression in mouse airway epithelium. J. Virol. 72, 7302-7309.

Schmidt, B. Z., Haaf, J. B., Leal, T., and Noel, S. (2016). Cystic fibrosis transmembrane conductance regulator modulators in cystic fibrosis: current perspectives. Clin. Pharmacol. Adv. Appl. 8, 127-140. doi: 10.2147/CPAA. S100759

Schwank, G., Koo, B.-K., Sasselli, V., Dekkers, J. F., Heo, I., Demircan, T., et al. (2013). Functional repair of CFTR by CRISPR/Cas9 in intestinal stem cell organoids of cystic fibrosis patients. Cell Stem Cell 13, 653-658. doi: 10.1016/ j.stem.2013.11.002

Sermet-Gaudelus, I., Boeck, K. D., Casimir, G. J., Vermeulen, F., Leal, T., Mogenet, A., et al. (2010). Ataluren (PTC124) induces cystic fibrosis transmembrane conductance regulator protein expression and activity in children with nonsense mutation cystic fibrosis. Am. J. Respir. Crit. Care Med. 182, 1262-1272. doi: 10.1164/rccm.201001-0137OC

Sermet-Gaudelus, I., Renouil, M., Fajac, A., Bidou, L., Parbaille, B., Pierrot, S., et al. (2007). In vitro prediction of stop-codon suppression by intravenous gentamicin in patients with cystic fibrosis: a pilot study. BMC Med. 5:5. doi: 10.1186/1741-7015-5-5

Sharma, M., Pampinella, F., Nemes, C., Benharouga, M., So, J., Du, K., et al. (2004). Misfolding diverts CFTR from recycling to degradation: quality control at early endosomes. J. Cell Biol. 164, 923-933. doi: 10.1083/jcb.200312018

Simsek, S., Zhou, T., Robinson, C. L., Tsai, S.-Y., Crespo, M., Amin, S., et al. (2016). Modeling cystic fibrosis using pluripotent stem cell-derived human pancreatic ductal epithelial cells. Stem Cells Transl. Med. 5, 572-579. doi: 10.5966/sctm. 2015-0276

Sinha, C., Zhang, W., Moon, C. S., Actis, M., Yarlagadda, S., Arora, K., et al. (2015). Capturing the direct binding of CFTR correctors to CFTR by using click chemistry. ChemBioChem 16, 2017-2022. doi: 10.1002/cbic.2015 00123

Sloane, P. A., and Rowe, S. M. (2010). Cystic fibrosis transmembrane conductance regulator protein repair as a therapeutic strategy in cystic fibrosis. Curr. Opin. Pulm. Med. 16, 591-597. doi: 10.1097/MCP.0b013e32833f1d00

Stoltz, D. A., Meyerholz, D. K., and Welsh, M. J. (2015). Origins of cystic fibrosis lung disease. N. Engl. J. Med. 372, 351-362. doi: 10.1056/NEJMra1300109

Taylor-Cousar, J. L., Munck, A., McKone, E. F., van der Ent, C. K., Moeller, A., Simard, C., et al. (2017). Tezacaftor-ivacaftor in patients with cystic fibrosis homozygous for Phe508del. N. Engl. J. Med. 377, 2013-2023. doi: 10.1056/ NEJMoa1709846

Takahashi, K., Okita, K., Nakagawa, M., and Yamanaka, S. (2007). Induction of pluripotent stem cells from fibroblast cultures. Nat. Protoc. 2, 3081-3089. doi: 10.1038/nprot.2007.418

Thibodeau, P. H., Richardson, J. M., Wang, W., Millen, L., Watson, J., Mendoza, J. L., et al. (2010). The cystic fibrosis-causing mutation deltaF508 affects multiple steps in cystic fibrosis transmembrane conductance regulator biogenesis. J. Biol. Chem. 285, 35825-35835. doi: 10.1074/jbc.M110. 131623 
Van der Plas, S. E., Kelgtermans, H., De Munck, T., Martina, S. L. X., Dropsit, S., Quinton, E., et al. (2018). Discovery of N-(3-Carbamoyl-5,5,7,7-tetramethyl5,7-dihydro-4H-thieno[2,3-c]pyran-2-yl)-1H-pyrazole-5-carboxamide (GLPG1837), a novel potentiator which can open class III mutant cystic fibrosis transmembrane conductance regulator (CFTR) channels to a high extent. J. Med. Chem. 61, 1425-1435. doi: 10.1021/acs.jmedchem.7b01288

Van Goor, F., Hadida, S., Grootenhuis, P. D. J., Burton, B., Cao, D., Neuberger, T., et al. (2009). Rescue of CF airway epithelial cell function in vitro by a CFTR potentiator, VX-770. Proc. Natl. Acad. Sci. U.S.A. 106, 18825-18830. doi: 10. 1073/pnas.0904709106

Van Goor, F., Hadida, S., Grootenhuis, P. D. J., Burton, B., Stack, J. H., Straley, K. S., et al. (2011). Correction of the F508del-CFTR protein processing defect in vitro by the investigational drug VX-809. Proc. Natl. Acad. Sci. U.S.A. 108, 18843-18848. doi: 10.1073/pnas.1105787108

Van Goor, F., Hadida, S., Grootenhuis, P. D. J., Stack, J. H., Burton, B., Olson, E. R., et al. (2010). Rescue of the protein folding defect in cystic fibrosis in vitro by the investigational small molecule, VX-809. J. Cyst. Fibros. 9:S14. doi: 10.1016/S1569-1993(10)60050-1

Van Goor, F., Straley, K. S., Cao, D., González, J., Hadida, S., Hazlewood, A., et al. (2006). Rescue of DeltaF508-CFTR trafficking and gating in human cystic fibrosis airway primary cultures by small molecules. Am. J. Physiol. Lung Cell. Mol. Physiol. 290, L1117-L1130. doi: 10.1152/ajplung.00169.2005

Varga, K., Jurkuvenaite, A., Wakefield, J., Hong, J. S., Guimbellot, J. S., Venglarik, C. J., et al. (2004). Efficient intracellular processing of the endogenous cystic fibrosis transmembrane conductance regulator in epithelial cell lines. J. Biol. Chem. 279, 22578-22584. doi: 10.1074/jbc.M401522200

Veit, G., Avramescu, R. G., Chiang, A. N., Houck, S. A., Cai, Z., Peters, K. W., et al. (2016). From CFTR biology toward combinatorial pharmacotherapy: expanded classification of cystic fibrosis mutations. Mol. Biol. Cell 27, 424-433. doi: 10.1091/mbc.E14-04-0935

Veit, G., Avramescu, R. G., Perdomo, D., Phuan, P.-W., Bagdany, M., Apaja, P. M., et al. (2014). Some gating potentiators, including VX-770, diminish $\triangle$ F508-CFTR functional expression. Sci. Transl. Med. 6:246ra97. doi: 10.1126/ scitranslmed.3008889

Veit, G., Xu, H., Dreano, E., Avramescu, R. G., Bagdany, M., Beitel, L. K., et al. (2018). Structure-guided combination therapy to potently improve the function of mutant CFTRs. Nat. Med. 24, 1732-1742. doi: 10.1038/s41591-018$0200-\mathrm{x}$

Vergani, P., Lockless, S. W., Nairn, A. C., and Gadsby, D. C. (2005). CFTR channel opening by ATP-driven tight dimerization of its nucleotide-binding domains. Nature 433, 876-880. doi: 10.1038/nature03313

Vidović, D., Carlon, M. S., da Cunha, M. F., Dekkers, J. F., Hollenhorst, M. I., Bijvelds, M. J. C., et al. (2015). rAAV-CFTR $\Delta$ R rescues the cystic fibrosis phenotype in human intestinal organoids and cystic fibrosis mice. Am. J. Respir. Crit. Care Med. 193, 288-298. doi: 10.1164/rccm.201505-0914OC

Wagner, D. E., Cardoso, W. V., Gilpin, S. E., Majka, S., Ott, H., Randell, S. H., et al. (2016). An official American thoracic society workshop report 2015. Stem cells and cell therapies in lung biology and diseases. Ann. Am. Thorac. Soc. 13, S259-S278. doi: 10.1513/AnnalsATS.201502-086ST

Wainwright, C. E., Elborn, J. S., and Ramsey, B. W. (2015). Lumacaftor-ivacaftor in patients with cystic fibrosis homozygous for Phe508del CFTR. N. Engl. J. Med. 373, 1783-1784. doi: 10.1056/NEJMc1510466

Walters, R. W., Grunst, T., Bergelson, J. M., Finberg, R. W., Welsh, M. J., and Zabner, J. (1999). Basolateral localization of fiber receptors limits adenovirus infection from the apical surface of airway epithelia. J. Biol. Chem. 274, 1021910226. doi: $10.1074 /$ jbc.274.15.10219

Wang, W., Bernard, K., Li, G., and Kirk, K. L. (2007). Curcumin opens cystic fibrosis transmembrane conductance regulator channels by a novel mechanism that requires neither ATP binding nor dimerization of the nucleotidebinding domains. J. Biol. Chem. 282, 4533-4544. doi: 10.1074/jbc.M60994 2200

Wang, X., Liu, B., Searle, X., Yeung, C., Bogdan, A., Greszler, S., et al. (2018). Discovery of 4 - $\left[\left(\begin{array}{lll}2 & R, 4 & R\end{array}\right)-4\right.$ - $(\{[1-(2,2-$ Difluoro-1,3-benzodioxol5-yl)cyclopropyl]carbonyl\}amino)-7-(difluoromethoxy)-3,4-dihydro-2 $H$-chromen-2-yl]benzoic Acid (ABBV/GLPG-2222), a potent cystic fibrosis transmembrane conductance regulator (CFTR) corrector for the treatment of cystic fibrosis. J. Med. Chem. 61, 1436-1449. doi: 10.1021/acs.jmedchem.7b01339
Wang, X., Venable, J., LaPointe, P., Hutt, D. M., Koulov, A. V., Coppinger, J., et al. (2006). Hsp90 cochaperone Ahal downregulation rescues misfolding of CFTR in cystic fibrosis. Cell 127, 803-815. doi: 10.1016/j.cell.2006. 09.043

Weinreich, F., Wood, P. G., Riordan, J. R., and Nagel, G. (1997). Direct action of genistein on CFTR. Pflügers Arch. 434, 484-491. doi: 10.1007/s004240050424

Welch, E. M., Barton, E. R., Zhuo, J., Tomizawa, Y., Friesen, W. J., Trifillis, P., et al. (2007). PTC124 targets genetic disorders caused by nonsense mutations. Nature 447, 87-91. doi: 10.1038/nature05756

Wellhauser, L., Kim Chiaw, P., Pasyk, S., Li, C., Ramjeesingh, M., and Bear, C. E. (2009). A small-molecule modulator interacts directly with deltaPhe508-CFTR to modify its ATPase activity and conformational stability. Mol. Pharmacol. 75, 1430-1438. doi: 10.1124/mol.109.055608

White, M. K., Kaminski, R., Young, W.-B., Roehm, P. C., and Khalili, K. (2017). CRISPR editing technology in biological and biomedical investigation. J. Cell. Biochem. 118, 3586-3594. doi: 10.1002/jcb.26099

Wilschanski, M., Famini, C., Blau, H., Rivlin, J., Augarten, A., Avital, A., et al. (2000). A pilot study of the effect of gentamicin on nasal potential difference measurements in cystic fibrosis patients carrying stop mutations. Am. J. Respir. Crit. Care Med. 161, 860-865. doi: 10.1164/ajrccm.161.3.9904116

Wilschanski, M., Miller, L. L., Shoseyov, D., Blau, H., Rivlin, J., Aviram, M., et al. (2011). Chronic ataluren (PTC124) treatment of nonsense mutation cystic fibrosis. Eur. Respir. J. 38, 59-69. doi: 10.1183/09031936.00120910

Wilschanski, M., Yahav, Y., Yaacov, Y., Blau, H., Bentur, L., Rivlin, J., et al. (2003). Gentamicin-induced correction of CFTR function in patients with cystic fibrosis and CFTR stop mutations. N. Engl. J. Med. 349, 1433-1441. doi: 10.1056/NEJMoa022170

Xue, X., Mutyam, V., Tang, L., Biswas, S., Du, M., Jackson, L. A., et al. (2014). Synthetic aminoglycosides efficiently suppress cystic fibrosis transmembrane conductance regulator nonsense mutations and are enhanced by ivacaftor. Am. J. Respir. Cell Mol. Biol. 50, 805-816. doi: 10.1165/rcmb.2013-0282OC

Yan, Z., Feng, Z., Sun, X., Zhang, Y., Zou, W., Wang, Z., et al. (2017). Human bocavirus type- 1 capsid facilitates the transduction of ferret airways by adenoassociated virus genomes. Hum. Gene Ther. 28, 612-625. doi: 10.1089/hum. 2017.060

Yang, H., Shelat, A. A., Guy, R. K., Gopinath, V. S., Ma, T., Du, K., et al. (2003). Nanomolar affinity small molecule correctors of defective Delta F508-CFTR chloride channel gating. J. Biol. Chem. 278, 35079-35085. doi: 10.1074/jbc. M303098200

Yeh, H.-I., Sohma, Y., Conrath, K., and Hwang, T.-C. (2017). A common mechanism for CFTR potentiators. J. Gen. Physiol. 149, 1105-1118. doi: 10. 1085/jgp.201711886

Yu, G. J., Yoo, C. L., Yang, B., Lodewyk, M. W., Meng, L., El-Idreesy, T. T., et al. (2008). Potent s-cis-locked bithiazole correctors of DeltaF508 cystic fibrosis transmembrane conductance regulator cellular processing for cystic fibrosis therapy. J. Med. Chem. 51, 6044-6054. doi: 10.1021/jm800533c

Yu, H., Burton, B., Huang, C.-J., Worley, J., Cao, D., Johnson, J. P., et al. (2012). Ivacaftor potentiation of multiple CFTR channels with gating mutations. J. Cyst. Fibros. 11, 237-245. doi: 10.1016/j.jcf.2011.12.005

Yui, S., Nakamura, T., Sato, T., Nemoto, Y., Mizutani, T., Zheng, X., et al. (2012). Functional engraftment of colon epithelium expanded in vitro from a single adult Lgr5+ stem cell. Nat. Med. 18, 618-623. doi: 10.1038/ nm.2695

Zabner, J., Couture, L. A., Gregory, R. J., Graham, S. M., Smith, A. E., and Welsh, M. J. (1993). Adenovirus-mediated gene transfer transiently corrects the chloride transport defect in nasal epithelia of patients with cystic fibrosis. Cell 75, 207-216. doi: 10.1016/0092-8674(93)80063-K

Zabner, J., Ramsey, B. W., Meeker, D. P., Aitken, M. L., Balfour, R. P., Gibson, R. L., et al. (1996). Repeat administration of an adenovirus vector encoding cystic fibrosis transmembrane conductance regulator to the nasal epithelium of patients with cystic fibrosis. J. Clin. Invest. 97, 1504-1511. doi: 10.1172/ JCI118573

Zaman, K., Sawczak, V., Zaidi, A., Butler, M., Bennett, D., Getsy, P., et al. (2016). Augmentation of CFTR maturation by S-nitrosoglutathione reductase. Am. J. Physiol. Lung Cell. Mol. Physiol. 310, L263-L270. doi: 10.1152/ajplung.00269. 2014

Zamecnik, P. C., Raychowdhury, M. K., Tabatadze, D. R., and Cantiello, H. F. (2004). Reversal of cystic fibrosis phenotype in a cultured $\Delta 508$ cystic 
fibrosis transmembrane conductance regulator cell line by oligonucleotide insertion. Proc. Natl. Acad. Sci. U.S.A. 101, 8150-8155. doi: 10.1073/pnas.04019 33101

Zawistoski, M., Sui, J., Ordonez, C., Mai, V., Liu, E., Li, T., et al. (2016). 32 Properties of a novel F508del-CFTR corrector FDL169. J. Cyst. Fibros. 15, S59-S60. doi: 10.1016/S1569-1993(16) 30272-7

Zeitlin, P. L., Diener-West, M., Rubenstein, R. C., Boyle, M. P., Lee, C. K. K., and Brass-Ernst, L. (2002). Evidence of CFTR function in cystic fibrosis after systemic administration of 4-phenylbutyrate. Mol. Ther. 6, 119-126. doi: 10. 1006/mthe.2002.0639

Zhou, Z., Wang, X., Li, M., Sohma, Y., Zou, X., and Hwang, T.-C. (2005). High affinity ATP/ADP analogues as new tools for studying CFTR gating. J. Physiol. 569, 447-457. doi: 10.1113/jphysiol.2005. 095083
Conflict of Interest Statement: IS-G is principal investigator for studies funded by Vertex Pharmaceuticals, has received research fundings from Vertex Pharmaceuticals, and is part of the scientific Advisory board of Proteostasis Inc.

The remaining authors declare that the research was conducted in the absence of any commercial or financial relationships that could be construed as a potential conflict of interest.

Copyright $\odot 2019$ Pranke, Golec, Hinzpeter, Edelman and Sermet-Gaudelus. This is an open-access article distributed under the terms of the Creative Commons Attribution License (CC BY). The use, distribution or reproduction in other forums is permitted, provided the original author(s) and the copyright owner(s) are credited and that the original publication in this journal is cited, in accordance with accepted academic practice. No use, distribution or reproduction is permitted which does not comply with these terms. 